\title{
Pharmacological strategies to prevent postoperative delirium: a systematic review and network meta-analysis
}

\author{
Jun Mo Lee ${ }^{1}$, Ye Jin Cho' ${ }^{1}$, Eun Jin Ahn ${ }^{1}$, Geun Joo Choi ${ }^{1}$, and \\ Hyun Kang ${ }^{1,2}$
}

Received September 25, 2020

Revised October 15, 2020

Accepted October 16, 2020

\section{Corresponding author}

Hyun Kang, M.D., Ph.D.

Department of Anesthesiology and Pain Medicine, Chung-Ang University College of Medicine, 84 Heukseok-ro, Dongjak-gu, Seoul 06974, Korea Tel: 82-2-6299-2586

Fax: 82-2-6299-2585

E-mail: roman00@naver.com
${ }^{1}$ Department of Anesthesiology and Pain Medicine, Chung-Ang University College of Medicine, ${ }^{2}$ The Institute of Evidence Based Clinical Medicine, Chung-Ang University, Seoul, Korea

Background: Postoperative delirium (POD) is a condition of cerebral dysfunction and a common complication after surgery. This study aimed to compare and determine the relative efficacy of pharmacological interventions for preventing POD using a network meta-analysis.

Methods: We performed a systematic and comprehensive search to identify and analyze all randomized controlled trials until June 29, 2020, comparing two or more pharmacological interventions, including placebo, to prevent or reduce POD. The primary outcome was the incidence of POD. We performed a network meta-analysis and used the surface under the cumulative ranking curve (SUCRA) values and rankograms to present the hierarchy of the pharmacological interventions evaluated.

Results: According to the SUCRA value, the incidence of POD decreased in the following order: the combination of propofol and acetaminophen (86.1\%), combination of ketamine and dexmedetomidine (86.0\%), combination of diazepam, flunitrazepam, and pethidine $(84.8 \%)$, and olanzapine (75.6\%) after all types of anesthesia; combination of propofol and acetaminophen (85.9\%), combination of ketamine and dexmedetomidine (83.2\%), gabapentin (82.2\%), and combination of diazepam, flunitrazepam, and pethidine (79.7\%) after general anesthesia; and ketamine (87.1\%), combination of propofol and acetaminophen (86.0\%), and combination of dexmedetomidine and acetaminophen (66.3\%) after cardiac surgery. However, only the dexmedetomidine group showed a lower incidence of POD than the control group after all types of anesthesia and after general anesthesia.

Conclusions: Dexmedetomidine reduced POD compared with the control group. The combination of propofol and acetaminophen and the combination of ketamine and dexmedetomidine seemed to be effective in preventing POD. However, further studies are needed to determine the optimal pharmacological intervention to prevent POD.

Keywords: Delirium; Network meta-analysis; Pharmacology; Surgical procedures, operative.

This is an Open Access article distributed under the terms of the Creative Commons Attribution Non-Commercial License (http://creativecommons.org/licenses/by-nc/4.0) which permits unrestricted non-commercial use, distribution, and reproduction in any medium, provided the original work is properly cited.

Copyright (C) the Korean Society of Anesthesiologists, 2021 


\section{INTRODUCTION}

Postoperative delirium (POD) is a condition of cerebral dysfunction and a common complication after surgery that occurs in $15-35 \%$ patients [1]. Old age, a history of stroke, use of narcotic analgesics, poor physical condition, alcoholism, preexisting cognitive impairment, and type of surgery are known risk factors for POD [2,3]. Especially patients undergoing major surgery, including cardiac surgery, are at increased risk of developing POD because of the complexity of the surgical procedure, the administration of intraoperative and postoperative anesthetic and other pharmacological agents. For this reason, POD is reported to affect up to $57 \%$ of cardiac-surgery patients [4].

POD is characterized by altered consciousness, disorientation, impaired memory, perceptual disturbance, altered psychomotor activity, and altered sleep-wake cycles after surgery. POD increases the rate of mortality, length of hospital stay, risk of placement to long-term care institutions, or functional disability, thus increasing hospitalization costs $[2,5]$. Therefore, appropriate prevention and treatment of POD is important for enhancing postoperative recovery and quality of life in elderly patients [6].

The treatment strategies for POD are well organized compared to the prevention strategies. The treatment for POD includes treating the underlying cause; correcting fluid and electrolyte imbalance or hypoxia; removing catheters if present, and treating patients who are restless, aggressive, agitative, and harm to themself or others with antipsychotics such as haloperidol, chlorpromazine, olanzapine, and risperidone $[7,8]$.

However, it is unclear which strategies are effective for preventing POD. Therefore, various strategies to prevent POD, especially variable pharmacological interventions, such as dexmedetomidine, propofol, midazolam, ketamine, and acetaminophen, have been applied and compared. However, each study only compared two or three drugs and reported diverse results.

Recently, a few systematic reviews and meta-analyses have demonstrated and integrated the preventive effect of various interventions [9-14]. However, each study was limited to pair-wise meta-analysis and examined only two pharmacological interventions. No previous network meta-analysis (NMA) has compared the effectiveness of all available pharmacological interventions. Further, the aforementioned studies included studies conducted prior to 2017 .
NMA complements traditional pair-wise meta-analysis by combining direct and indirect comparisons of treatments and provides objective ranking of various treatments based on the corresponding surface under the cumulative ranking curve (SUCRA) [15].

Thus, we reviewed all articles that investigated the effectiveness of pharmacological interventions to prevent POD and performed NMA to compare and quantify the rank order of the effectiveness of pharmacological interventions to prevent POD.

\section{MATERIALS AND METHODS}

\section{Protocol and registration}

We developed the protocol for this systematic review and NMA according to the preferred reporting requirements for systematic review and meta-analysis protocol (PRISMA-P) statement [16]. We registered the review protocol at the

International Prospective Register of Systematic Reviews (registration no. CRD42020189363; www.crd.york.ac.uk/ prospero) on May 7, 2020.

This systematic review and NMA of pharmacological interventions for preventing POD were performed according to the protocol recommended by the Cochrane Collaboration [17] and reported according to the PRISMA extension for NMA guidelines [18].

\section{Search strategy}

We searched MEDLINE, EMBASE, Cochrane Central Register of Controlled Trials (CENTRAL), and Google Scholar from inception to June 29, 2020 using the search terms related to pharmacological interventions for preventing POD. The search terms used for MEDLINE and EMBASE are presented in Supplementary Material. Two investigators (GJC and HK) screened the titles and abstracts of the retrieved articles. Reference lists were imported to Endnote software 8.1 (Thompson Reuters, USA), and duplicate articles were removed. Additionally, the references of articles obtained from the original search were reviewed to identify relevant articles.

\section{Inclusion criteria and exclusion criteria}

We included only randomized controlled trials (RCTs) that compared two or more pharmacological interventions 
to prevent POD.

The PICO-SD information included the following:

1. Patients $(\mathrm{P})$ : all patients receiving surgery under general or regional anesthesia

2. Intervention (I): pharmacological interventions to prevent POD

3. Comparison (C): other pharmacological interventions to prevent POD, placebo, or no treatment

4. Outcome measurements $(\mathrm{O})$ : the incidence of POD

5. Study design (SD): RCTs

6. Subgroup analysis: general anesthesia and cardiac surgery

Exclusion criteria contained the following features:

1. Review articles, case reports, case-series, letters to the editor, commentaries, proceedings, laboratory science studies, and all other non-relevant studies

2. Studies that failed to report the outcomes of interest

3. Studies that investigated the effect of inhalational anesthetics or patient-controlled analgesia (PCA) regimens

There was on language limitations or date restrictions in our study.

\section{Study selection}

Two reviewers (JML and YJC) independently screened the titles and abstracts of the studies to identify trials that met the inclusion criteria outlined above. For articles determined to be eligible based on the title and/or abstract, the full paper was retrieved. Potentially relevant studies chosen by at least one author were retrieved, and the full text was evaluated. Full-text articles were assessed separately by two authors (JML and YJC), and any disagreements were resolved through discussion. In cases where agreement could not be reached, the dispute was resolved with the help of a third investigator (HK). To minimize data duplication owing to multiple reporting, articles from the same author, organization, or country were compared.

\section{Data extraction}

Using a standardized extraction form, the following data were extracted independently by two investigators (JML and YJC): 1) title, 2) name of the first author, 3) name of the journal, 4) year of publication, 5) study design, 6) type of pharmacological interventions, 7) dose of pharmacological agents, 8) country, 9) risk of bias, 10) inclusion criteria, 11) exclusion criteria, 12) age, 13) number of subjects, and 14) incidence of POD.

If the information was inadequate, attempts were made to contact the study authors, and additional information was requested. If unsuccessful, missing information was calculated from the available data, if possible, or was extracted from the figure using the open source software Plot Digitizer (version 2.6.8; http://plotdigitizer.sourceforge. net).

The reference lists were divided into two halves. Two investigators completed data extraction, one for each half of the reference list. Data extraction forms were crosschecked to verify the accuracy and consistency of the extracted data.

The degree of agreement between the two independent data extractors was computed using kappa statistics to measure the difference between the observed and expected agreements, i.e., whether they were random or by chance. Kappa values were interpreted as: 1) less than 0: less than chance agreement; 2) 0.01 to 0.20 : slight agreement; 3) 0.21 to 0.40 : fair agreement; 4) 0.41 to 0.60 : moderate agreement; 5) 0.61 to 0.80 : substantial agreement; and 6) 0.8 to 0.99: almost perfect agreement [19].

\section{Risk of bias assessment}

The quality of the studies was independently assessed by two investigators (JML and YJC) using the Revised Cochrane risk of bias tool for randomized trials (RoB 2.0). Risk of bias judgment was assessed in the following domains: bias arising from the randomization process, bias due to deviations from intended intervention, bias due to missing outcome data, bias in measurement of the outcome, and bias in selection of the reported results. Based on the results of risk of bias judgment, formal overall risk of bias judgment was categorized as "low risk of bias," "some concern," and "high risk of bias" [20].

\section{Statistical analysis}

Ad-hoc tables were designed to summarize data from the included studies by showing their key characteristics and any important questions related to the review objectives. After extracting the data, the reviewers determined the feasibility of a meta-analysis.

A multiple treatment comparison NMA is a meta-analysis generalization method that includes both direct and in- 
direct RCT comparison of treatments. A random-effects NMA based on a frequentist framework was performed using STATA software (version 15, StataCorp LP, USA) based on mvmeta with NMA graphical tools developed by Chaimani et al. [21].

Before conducting the NMA, we evaluated the transitivity assumption by examining the comparability of the risk of bias (all versus removing high risks of bias from the randomization process and overall risk of bias), demographics, and types of pharmacological interventions as potential treatment-effect modifiers across comparisons.

A network plot linking the included pharmacological interventions to prevent POD and their combination with other pharmacological agents was formed to indicate the types of agents, number of patients on different agents, and the level of pair-wise comparisons. The nodes show comparisons of pharmacological agents being compared, and the edges show the available direct comparisons among the pharmacological agents. The nodes and edges are weighed on the basis of the weights applied in NMA and the inverse of the standard error of effect.

We evaluated the consistency assumption for the entire network using the design-by-treatment interaction model. We also evaluated each closed loop in the network to evaluate local inconsistencies between the direct and indirect effect estimates for the same comparison. For each loop, we estimated the inconsistency factor (IF) as the absolute difference between the direct and indirect estimates and 95\% confidence interval (CI) for each paired comparison in the loop [22]. When the IF value with $95 \%$ CI started at 0 , it indicated that the direct evidence and the indirect evidence were consistent.

Mean summary effects with CI were presented together with their predictive intervals (PrIs) to facilitate interpretation of the results considering the magnitude of heterogeneity. PrIs provide an interval that is expected to encompass the estimate of a future study.

A rankogram and a cumulative ranking curve were drawn for each pharmacological intervention. Rankogram plots are the probabilities for treatments to assume a possible rank. We used surface under the cumulative ranking curve (SUCRA) values to present the hierarchy of pharmacological interventions to prevent the incidence of POD. The SUCRA is a relative ranking measure that accounts for the uncertainty in the treatment order, that is, accounts for both the location and the variance of all relative treatment effects. A higher SUCRA value is regarded as a more posi- tive result for individual interventions [23].

We performed subgroup analysis based on all types of anesthesia, general anesthesia, and cardiac surgery, because the incidence of POD is expected to be different according to the type of anesthesia, and increase after cardiac surgery.

\section{Quality of evidence}

The evidence grade was determined using the Grading of Recommendations, Assessment, Development, and Evaluation (GRADE) system, which uses a sequential assessment of the evidence quality, followed by an assessment of the risk-benefit balance and a subsequent judgment on the strength of the recommendations [24].

\section{RESULTS}

\section{Study selection}

We initially retrieved 235 articles from MEDLINE, EMBASE, CENTRAL, and Google Scholar databases and 17 articles through a manual search. After adjusting for duplicates, 245 studies remained. Of these, 182 studies were discarded after reviewing the title and abstracts for the following reasons: related to other topics, designed as systematic reviews, reviews or retrospective studies, and conference abstracts. The full texts of the 63 remaining studies were reviewed in detail; 12 studies were excluded for the following reasons: three were study protocols [25-27], two were editorials $[13,28]$, four were systematic reviews, [9-12] and three did not report the outcome of our interest (two compared an inhalational agent $[13,29]$ and one was compared in the PCA regimen [30]). Thus, 51 studies with a total of 22,565 patients that included 18 different pharmacological interventions were included in this NMA (Fig. 1). The kappa value for the selected articles between the two reviewers was 0.844 .

\section{Characteristics of the included studies}

The characteristics of the 51 studies are summarized in Table 1. All the studies were performed on adults with American Society of Anesthesiologists physical status classifications I, II, and III. The 51 studies were conducted in various countries, such as Australia [31,32], Canada [33,34], China [1,2,6,35-47], Denmark [48], Greece [49], India 


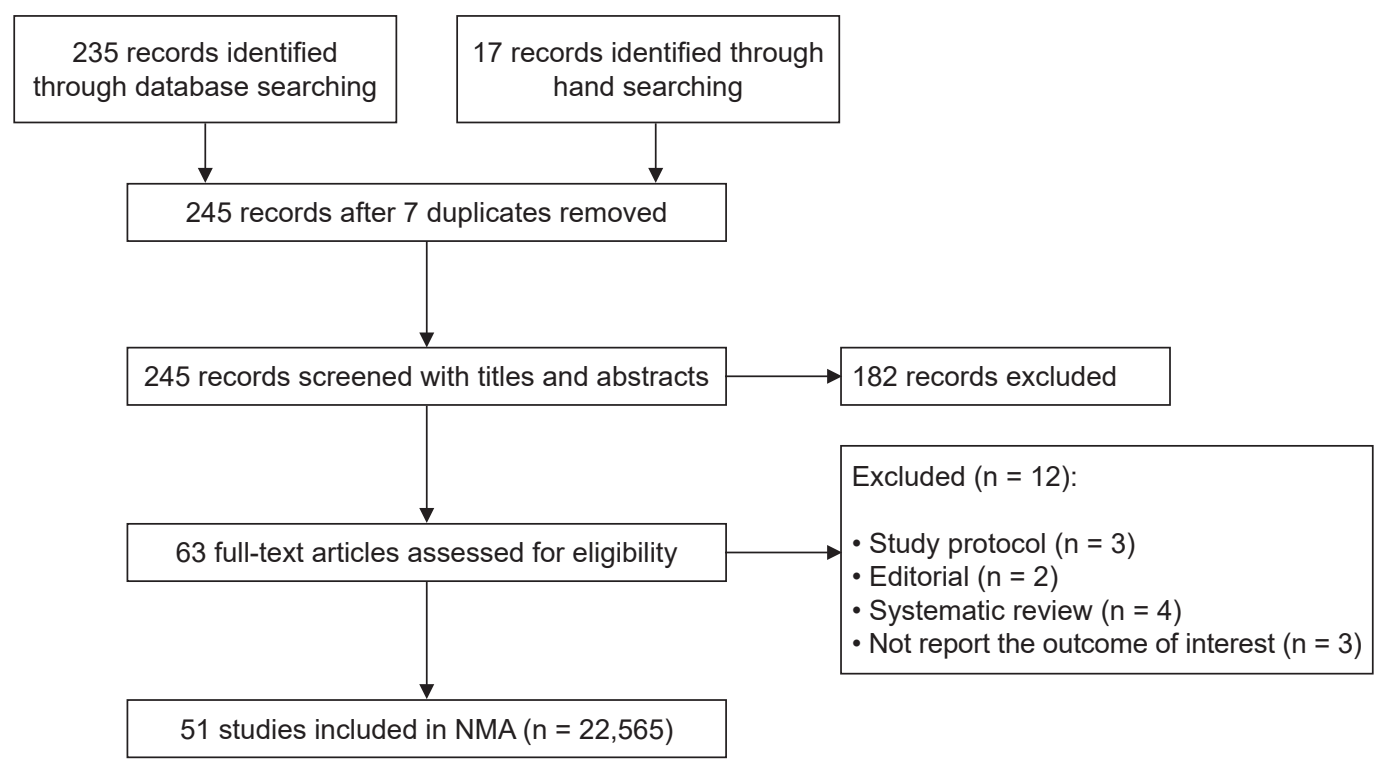

Fig. 1. PRISMA flowchart of included and excluded trials. PRISMA: preferred reporting requirements for systematic review and meta-analysis, NMA: network meta-analysis.

[50,51], Iran [52,53], Japan [54-57], the Netherlands [5861], South Korea [62,63], Switzerland [64], Taiwan [65,66], Thailand [67], the United Kingdom [68], the United States of America [4,69-76], and Saudi arabia [77].

Twenty-seven types of pharmacological agents, including dexmedetomidine (Dexm) $[2,4,6,31,33,35,36,38-45,47$, 50,51,62,63,65,66,69,70], propofol (Prop) [4,33,42-44, $47,51,66,70]$, acetaminophen (AAP) [70], midazolam (Mida) [4,6,77], remifentanil (Remi) [62], morphine (Morp) [31], methylprednisolone (MPDL) [32,34,48], melatonin (Mela) [58,77], dexamethasone (Dexa) [52,59,61], haloperidol (Halo) [37,54,55,60], rivastigmine (Riva) [64], ketamine (Keta) $[39,71]$, olanzapine (Olan) [72], gabapentin (Gaba) [73,76], nimodipine (Nimo) [1], cyproheptadine (Cypr) [53], ondansetron (Onda) [49], risperoidone (Risp) [67], L-tryptophan(L-tyr) [74], donepezil (Done) [68,75], Yokukansan (TJ-54) [56], diazepam (Diaz) [57], flunitrazepam (Flun) [57] and pethidine (Peth) [57], parecoxib (Pare) [46], and clonidine (Clon) [77] were evaluated.

The types of surgery investigated included cardiovascular surgery $[4,31-35,42,43,47,50-52,59,61,62,64,67,70,71]$, orthopedic surgery $[1,2,6,39,40,44,46,48,49,54,58,60,68,72,73,75-$ $77]$, thoracoscopic and pulmonary surgery $[41,56,65,74]$, abdominal and laparoscopic surgery [54-57,63,66], vascular and urology surgery [74], free flap [38], oral cancer surgery [45], and non-cardiac surgery $[36,37,53,69]$. The anesthesia method in the studies included only general anesthesia [1, 2,4,6,31-35,38,39,41-43,45,47,49-52,55-57,59,61-67,69$71,76]$, general anesthesia + regional anesthesia $[36,37,48$,
$54,72-74]$, type of anesthesia were not decribed $[40,53,58$, $60,68,75]$, and only regional anesthesia $[44,46,77]$.

\section{Study quality assessment}

The risk of bias assessment in the included studies using the Cochrane tool is presented in Table 2.

\section{All types of anesthesia}

A total of 51 studies $(22,565$ patients) measured the incidence of POD. The pooled overall incidence of POD after all types of anesthesia was $18.5 \%$ (95\% CI: $16.2 \%$ to $21.0 \%$, $\left.\mathrm{P}_{\text {chi }}{ }^{2}<0.001, \mathrm{I}^{2}=92.0 \%\right)$. The network plot of all eligible comparisons for this endpoint is depicted in Fig. 2A.

Although all 27 management modalities (nodes) were connected to the network, two comparisons (Control [Cont], Dexm) were compared directly to the other 25 nodes.

The evaluation of the network inconsistency using the design-by-treatment interaction model suggested no significant inconsistency $\left(\chi^{2}[8]=13.37, \mathrm{P}=0.100\right)$. Of the 14 closed loops in the network for the comparison of postoperative delirium, four loops (Dexm-Dexm + AAP-pro + AAP [01-04-05] [70], Dexm-Keta-Keta + Dexm [01-09-22] [39], Pro-Dexm + AAP-Prop + AAP [03-04-05] [70], Mida-Mela-Clon [06-11-25] [77]) were formed only by multi-arm trials. Thus, local inconsistency was evaluated in 10 loops. Although most loops showed no relevance in the local inconsistency between the direct and indirect point estimates, 


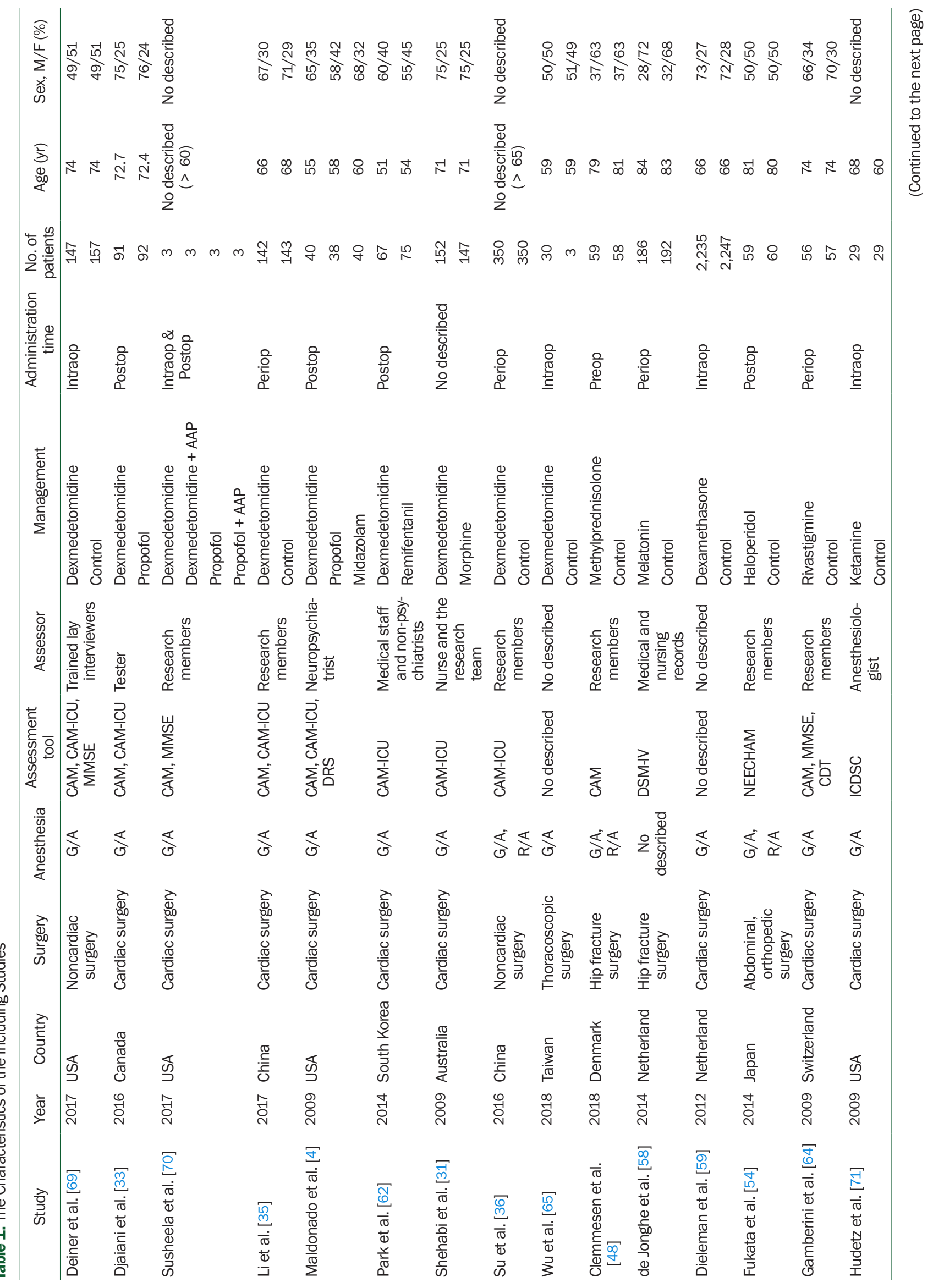




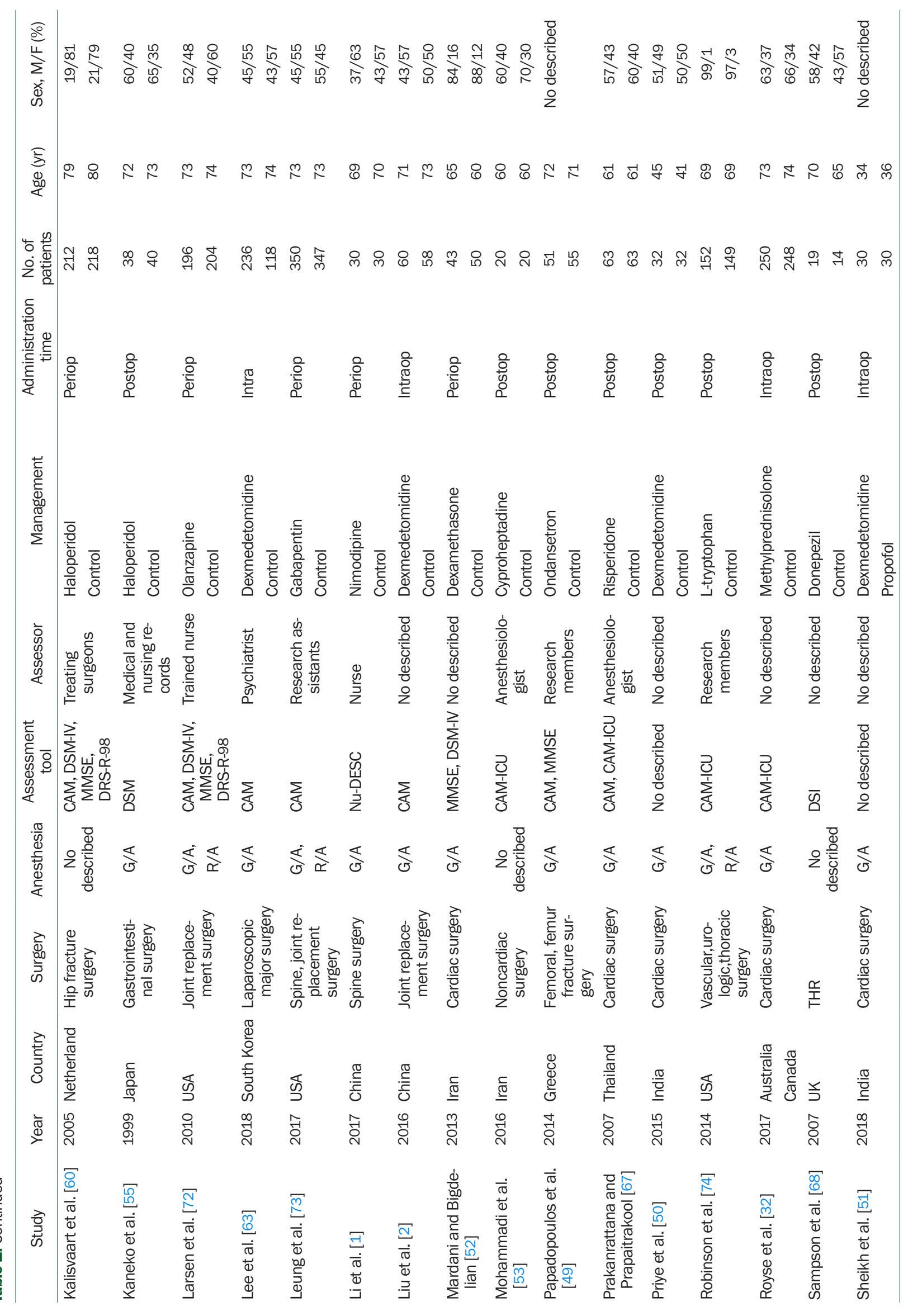




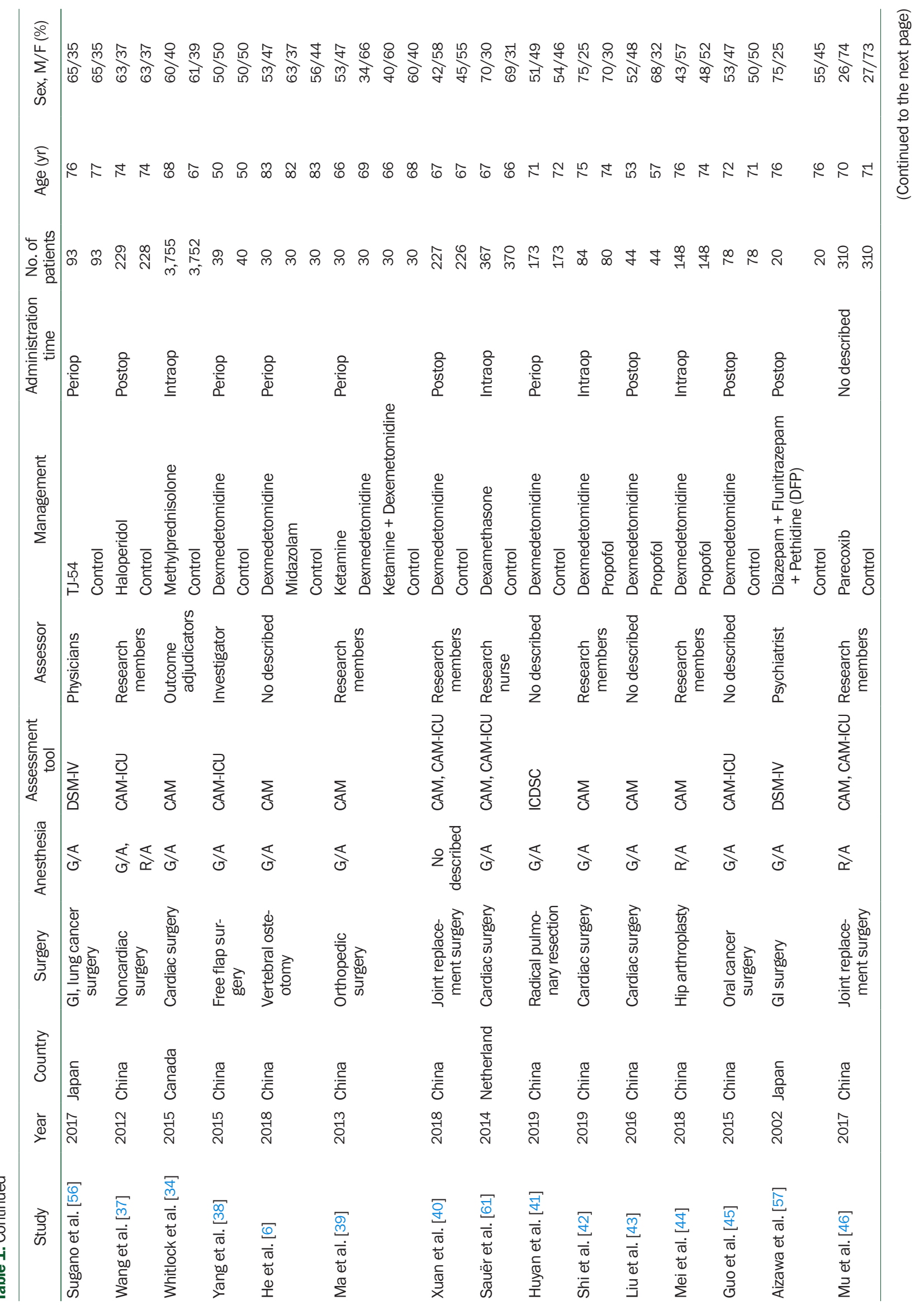




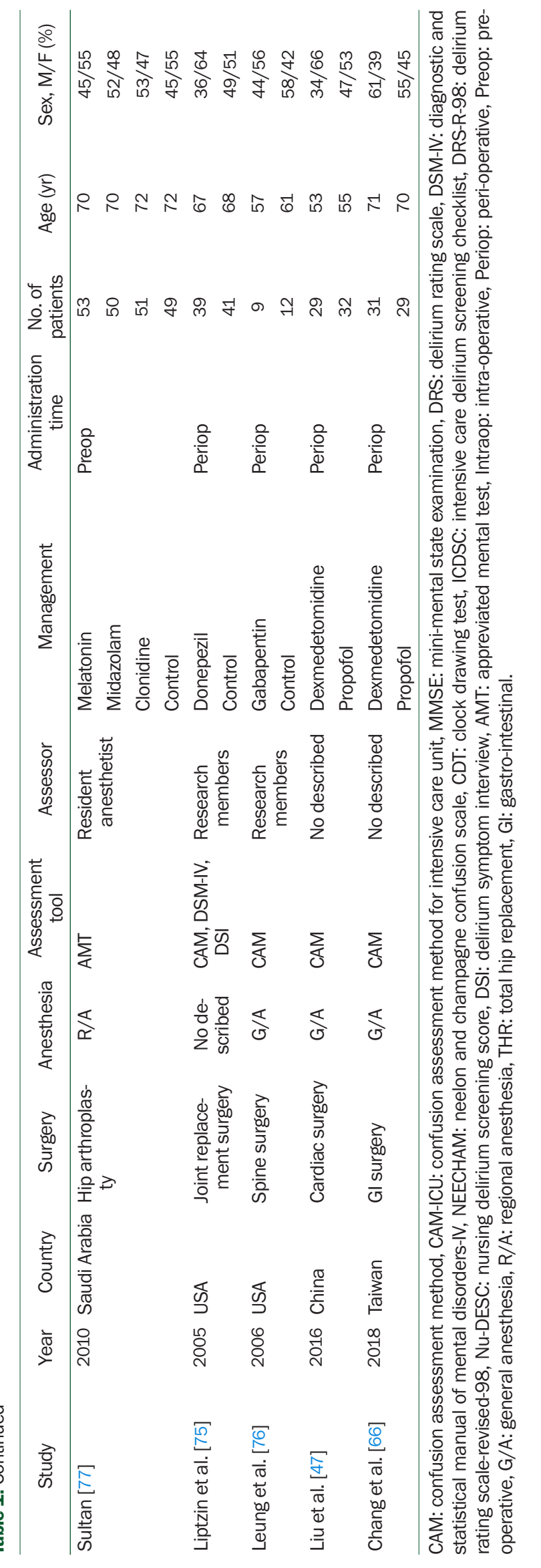

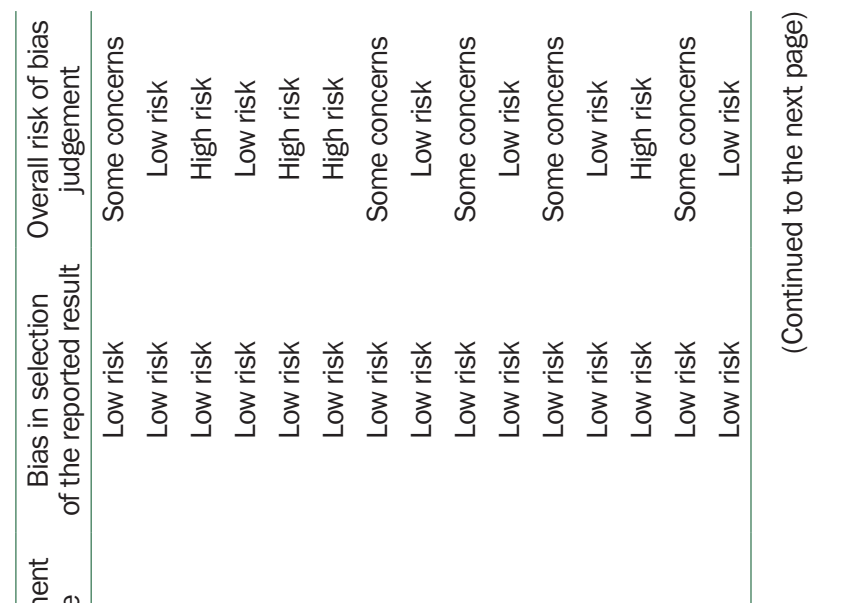

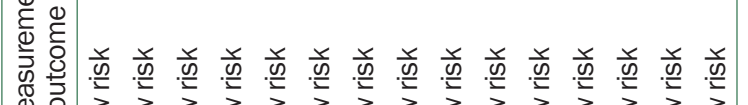

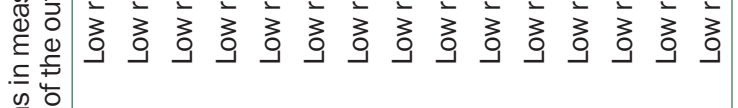

.

$\frac{\pi}{0}$

$\cdot \frac{\cos }{\overline{0}}$

है।

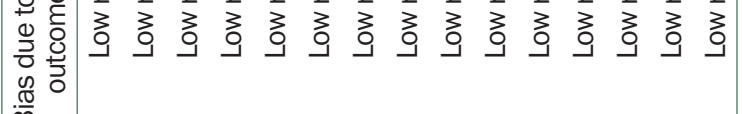

ह

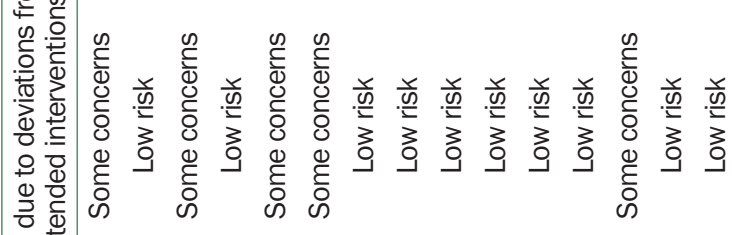

:.$\stackrel{ \pm}{.}$.

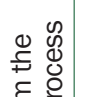

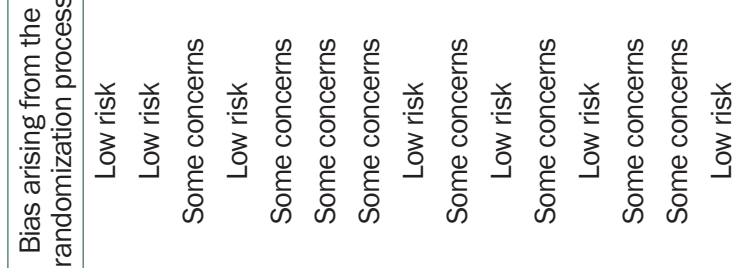

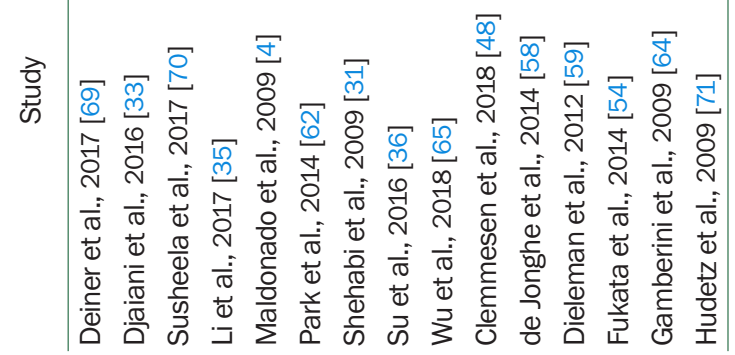




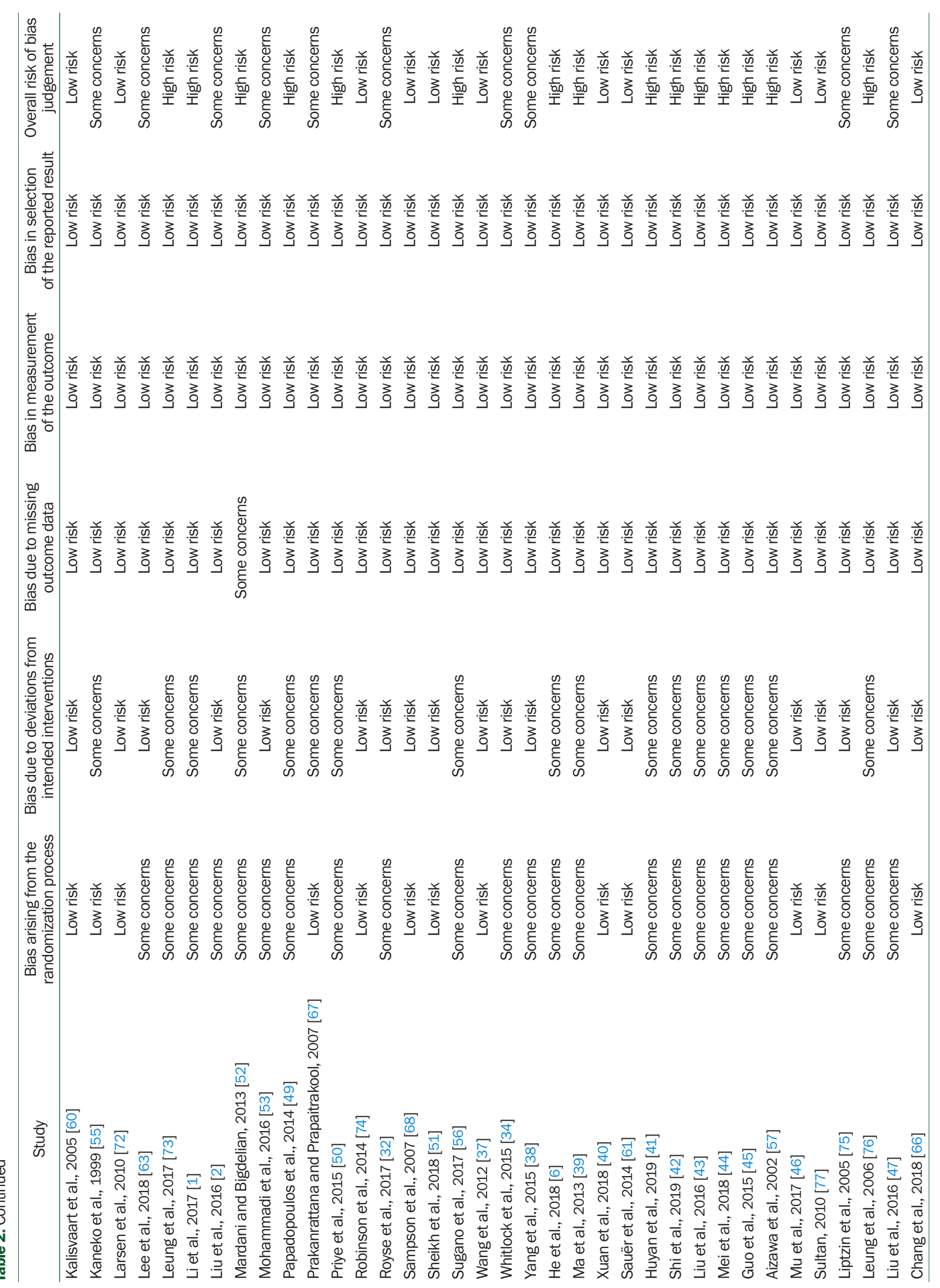



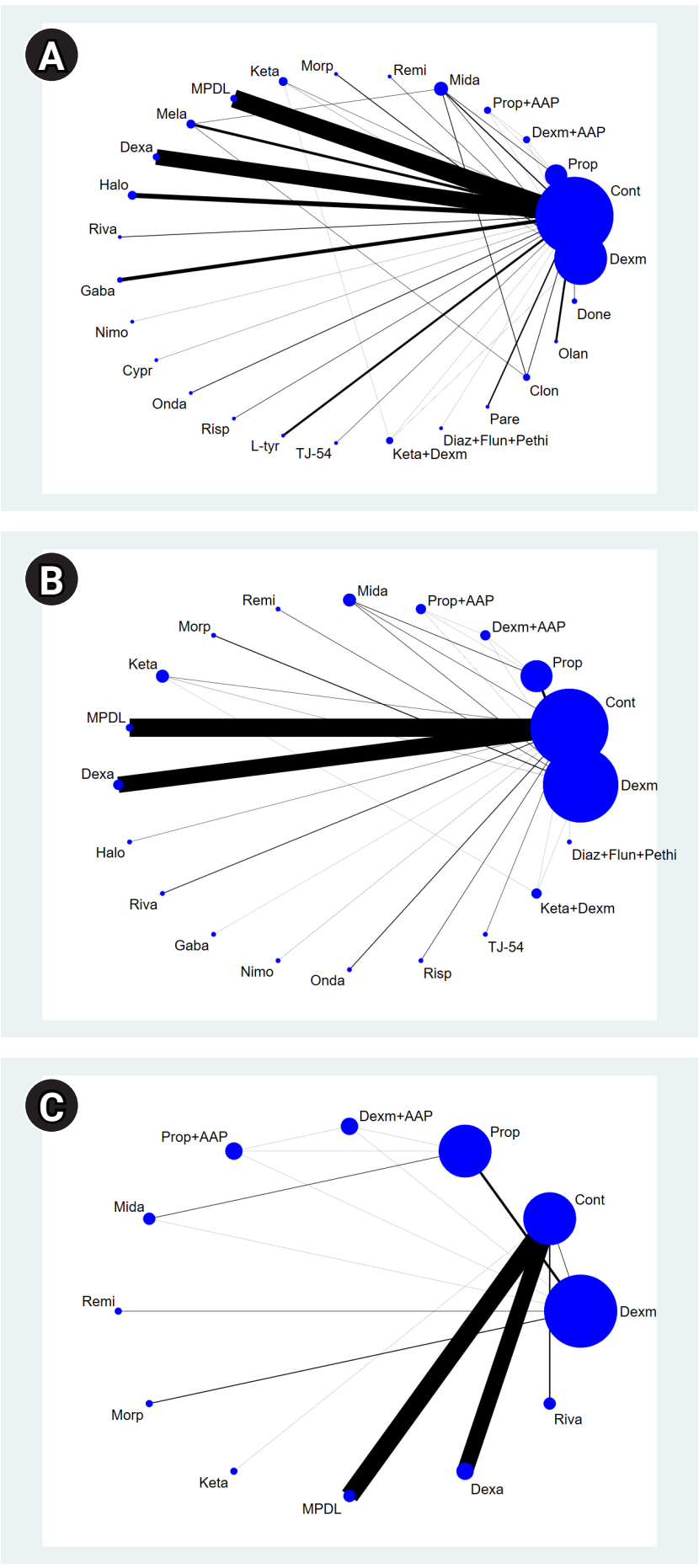

Fig. 2. Network plot of included studies comparing different pharmacological interventions. The nodes show a comparison of pharmacological interventions to prevent postoperative delirium, and the edges show the available direct comparisons among the pharmacological interventions. The nodes and edges are weighed on the basis of the weights applied in the network meta-analysis and the inverse of the standard error of effect. (A) All types of anesthesia, (B) general anesthesia, (C) cardiac surgery. inconsistencies were observed between the direct and indirect point estimates in the Cont-Mela-Clon (02-11-25) and Cont-Mida-Mela (02-06-11) loops (Fig. 3A).

Dexm showed a lower incidence of POD than Cont only in terms of $95 \%$ CI. Olan showed marginal significance compared with Cont in terms of 95\% CI (Fig. 4A). Insignificance in the $95 \%$ PrIs suggests that any future RCT could change the significance of the effectiveness of these comparisons.

The rankograms showed that Prop+AAP and Keta+Dexm had the lowest incidence of POD (Fig. 5A). The cumulative ranking plot was drawn, and the SUCRA probabilities of the different pharmacological agents for POD were calculated (Fig. 6A). The expected mean rankings and SUCRA values of each pharmacological intervention are presented in Fig. 7A. According to the SUCRA value, the incidence of POD was lower in the order of the Prop + AAP (86.1\%), followed by Keta + Dexm (86.0\%), Diaz + Flun + Pethi (84.8\%), and Olan (75.6\%). The comparison-adjusted funnel plots showed that the funnel plots were symmetrical around the zero line, which suggested a less likely publication bias (Fig. 8A).

\section{General anesthesia}

A total of 35 studies $(17,241$ patients) were analyzed. The pooled overall incidence of POD after general anesthesia was $16.5 \%$ (95\% CI: $14.2 \%$ to $19.2 \%, \mathrm{P}_{\text {chi }}{ }^{2}<0.001, \mathrm{I}^{2}=$ $89.3 \%)$.

The network plot of all eligible comparisons for this endpoint is depicted in Fig. 2B. Although all 20 management modalities (nodes) were connected to the network, two comparisons (Cont, Dexm) were directly compared to the other 18 nodes.

The evaluation of the network inconsistency using the design-by-treatment interaction model suggested no significant inconsistency $\left(\chi^{2}[6]=11.50, \mathrm{P}=0.074\right)$. Of the 10 closed loops in the network for the comparison of postoperative delirium, three loops (Dexm-Dexm + AAP-Prop + AAP [01-04-05] [70], Pro-Dexm + AAP-Prop + AAP [01-0919] [70], Dexm-Keta-Dexm + Keta [03-04-05] [39]) were formed only by multi-arm trials. Thus, local inconsistency was evaluated in seven loops. There was no significance in the local inconsistency between the direct and indirect point estimates (Fig. 3B).

Dexm showed a lower incidence of POD than Cont only in terms of 95\% CI (Fig. 4B). Insignificance in the 95\% PrIs suggests that any future RCT could change the significance 


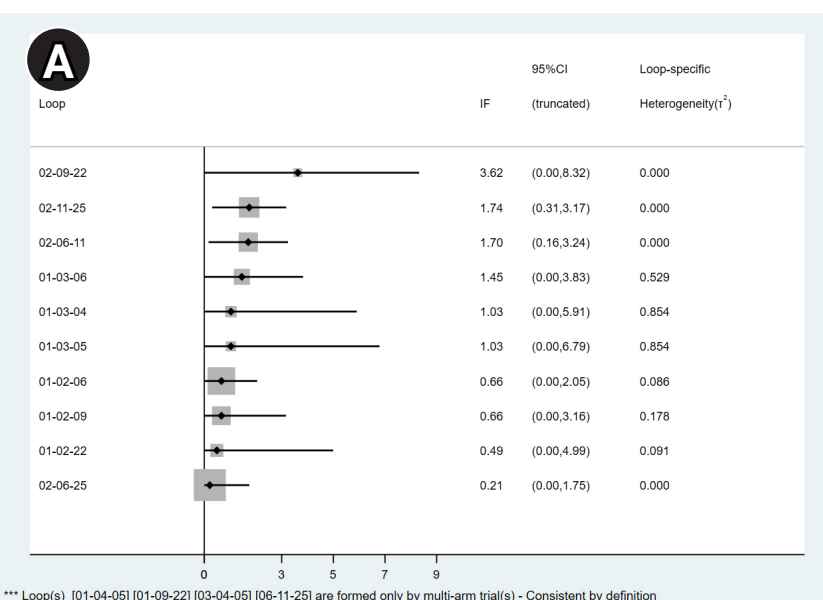

"." Loop(s) [01-04-05] [01-09-22] [03-04-05] [06-11-25] are formed only by multi-arm trial(s) - Consistent by definition
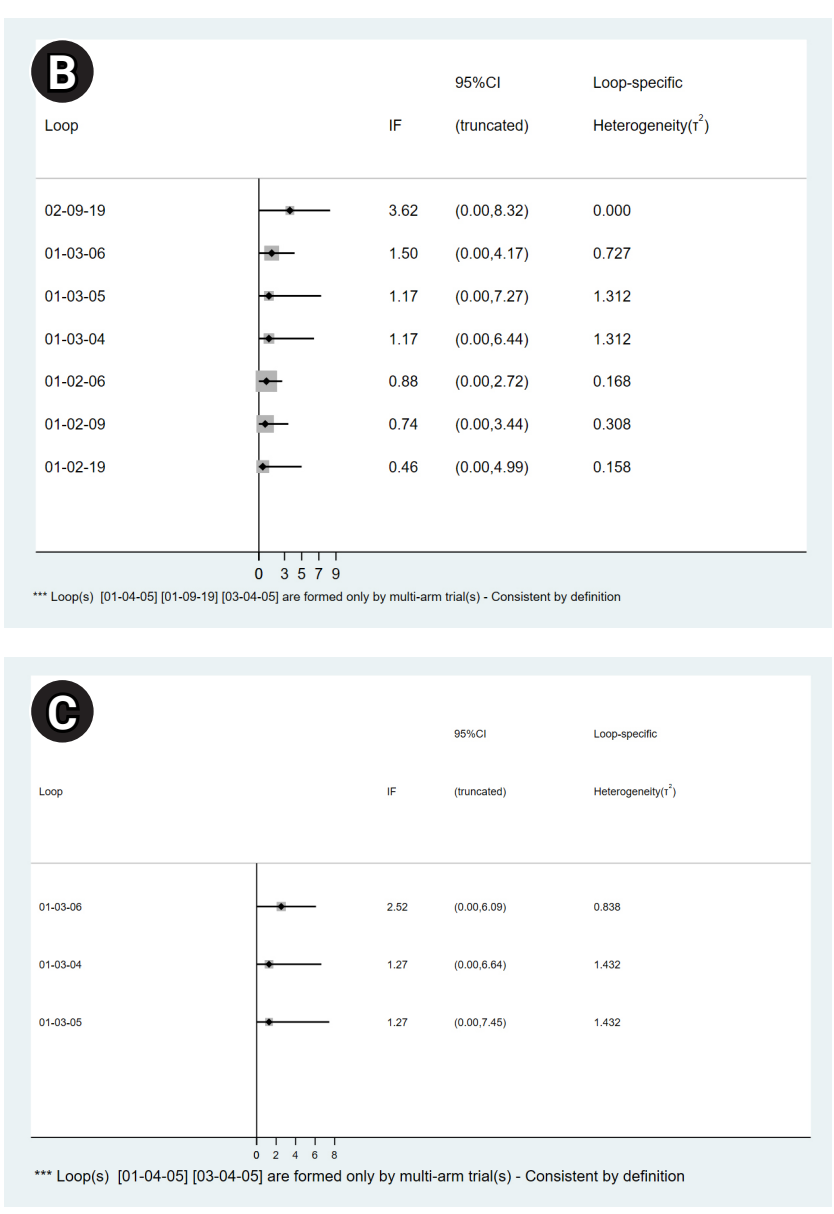

Fig. 3. Inconsistency plot between the direct and indirect effect estimates for the same comparison. Inconsistency factor (IF) as the absolute difference with 95\% confidence interval $(\mathrm{Cl})$ between the direct and indirect estimates for each paired comparison is presented. IF values close to 0 indicate that the two sources are in agreement. (A) All type of anesthesia, (B) general anesthesia, (C) cardiac surgery.
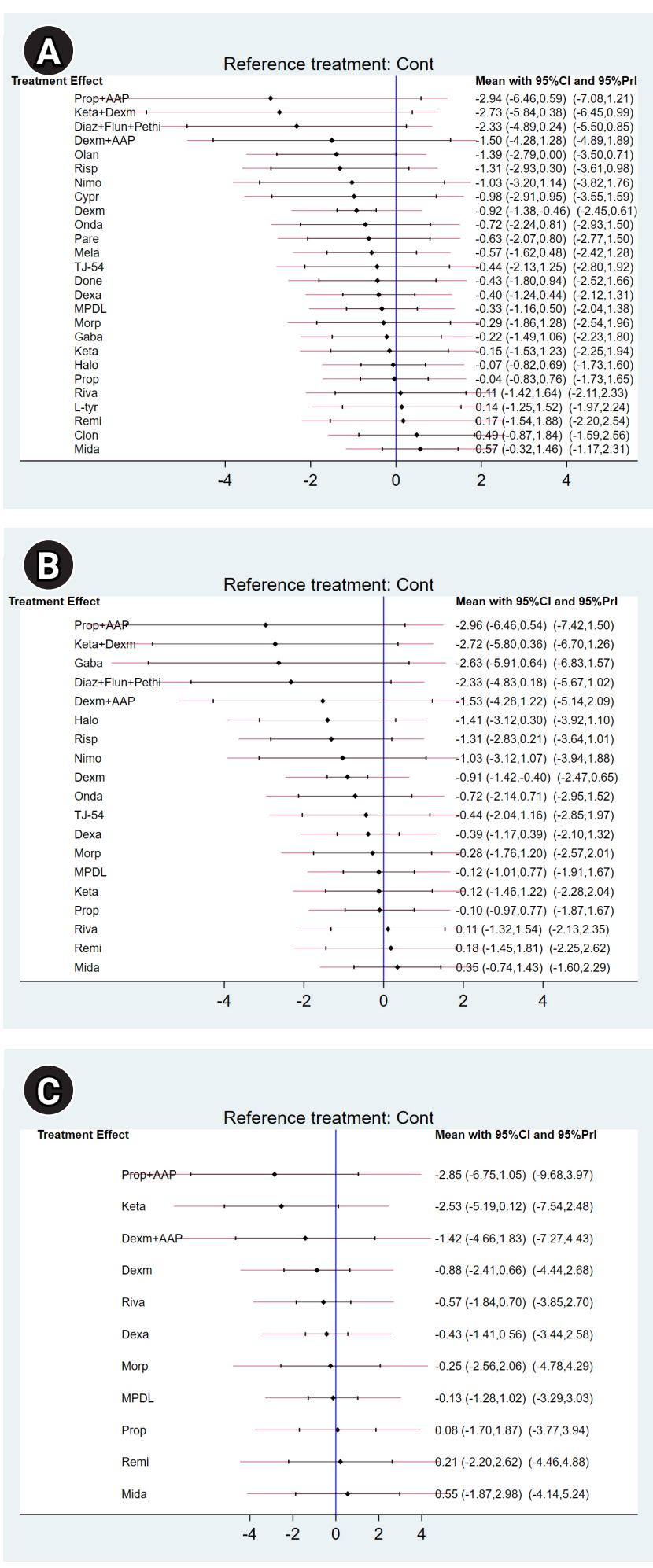

Fig. 4. Predictive interval plots between each management modality and placebo group. Diamond shape represents the mean summary effects. Black line represents the 95\% confidence interval (Cl), and red line represents the predictive interval ( $\mathrm{Prl})$. Prls provide an interval that is expected to encompass the estimate of a future study. (A) All type of anesthesia, (B) general anesthesia, (C) cardiac surgery. 


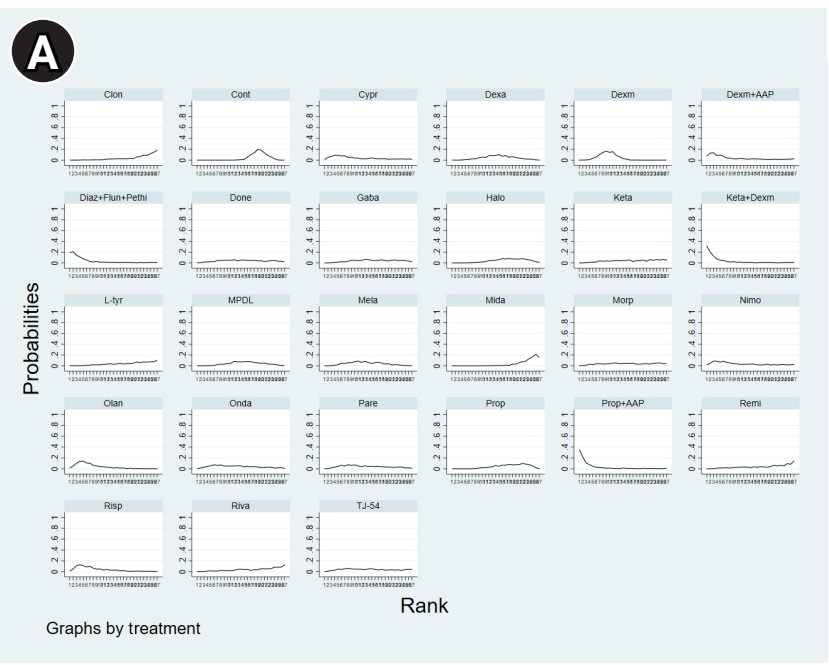

B

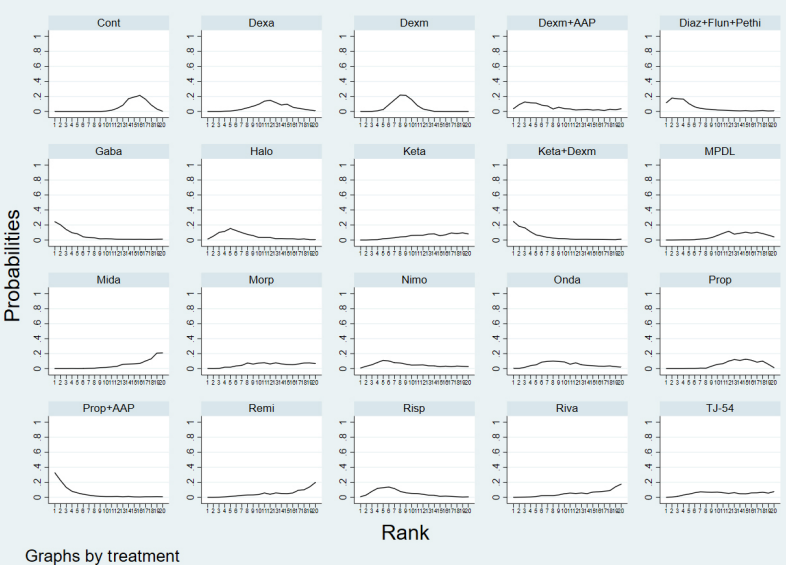

Graphs by treatment
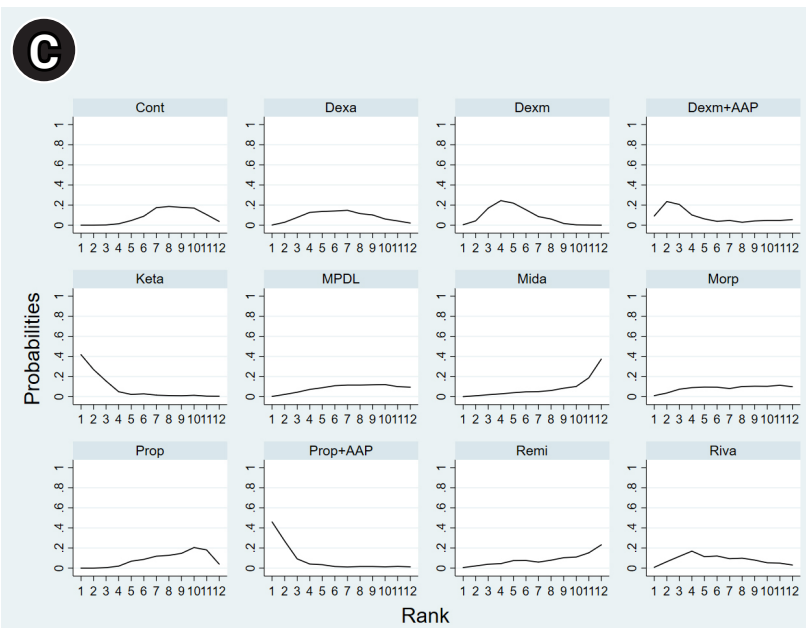

Graphs by treatment

Fig. 5. Rankogram. Profiles indicate the probabilities for treatments to assume any of the possible ranks. It is the probability that a given treatment ranks first, second, third, and so on, among all of the treatments evaluated in the NMA. (A) All type of anesthesia, (B) general anesthesia, (C) cardiac surgery. NMA: network metaanalysis.

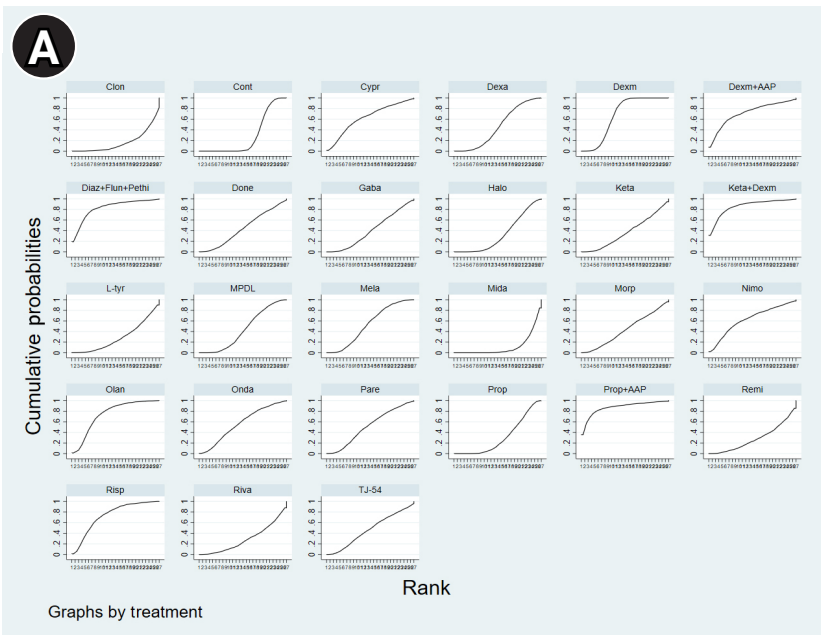

B
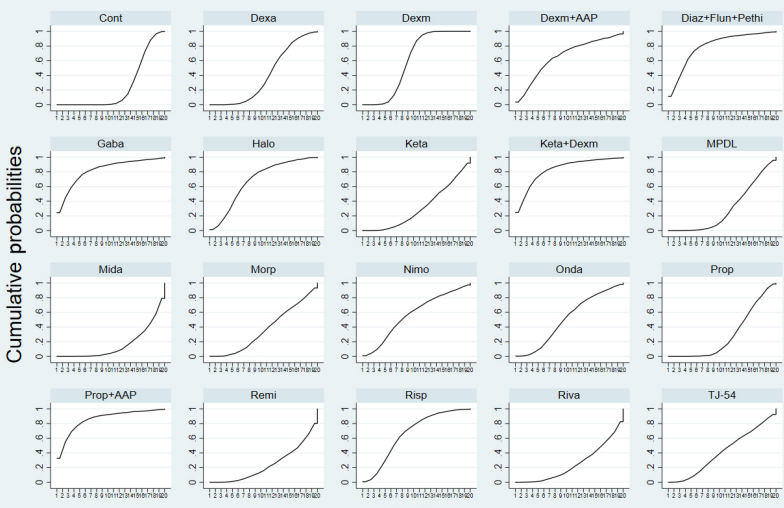

Graphs by treatment
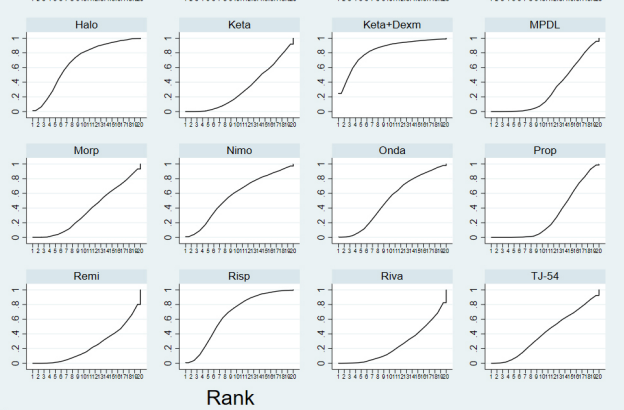

\section{C}

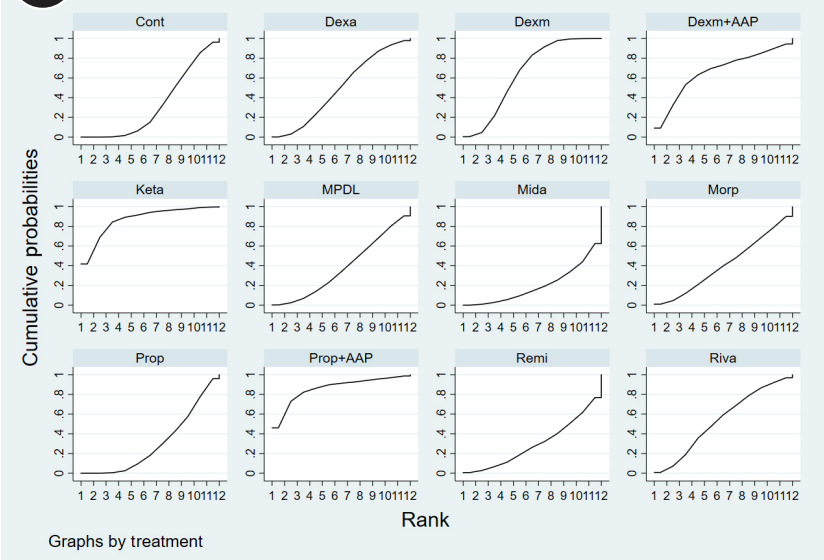

Fig. 6. Cumulative ranking curve plot. The profile indicates the sum of the probabilities from those ranked first, second, third, and so on. A higher cumulative ranking curve (surface of under cumulative ranking curve [SUCRA]) value is regarded as an improved result for an individual's intervention. When ranking treatments, the closer the SUCRA value is to $100 \%$, the higher the treatment ranking is relative to all other treatments. (A) All type of anesthesia, (B) general anesthesia, $(\mathrm{C})$ cardiac surgery. 


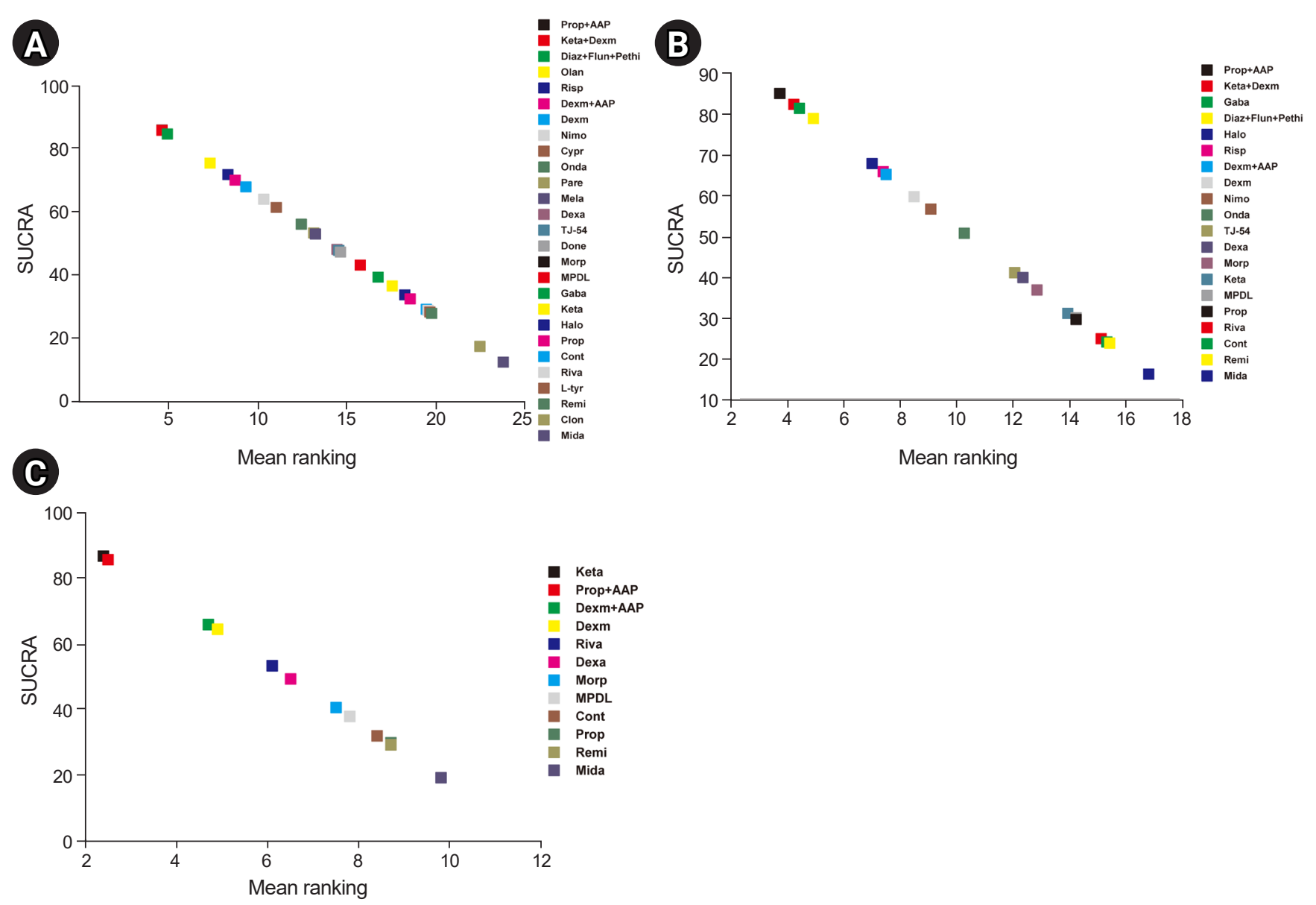

Fig. 7. Expected mean ranking and surface of under cumulative ranking curve (SUCRA) values. X-axis corresponds to expected mean ranking based on SUCRA value, and Y-axis corresponds to SUCRA value. (A) All type of anesthesia, (B) general anesthesia, (C) cardiac surgery.

of the effectiveness of these comparisons.

The rankogram showed that Prop + AAP, Keta + Dexm, and Gaba had the lowest incidence of POD (Fig. 5B). The cumulative ranking plot was drawn, and the SUCRA probabilities of the different pharmacological agents for the POD were calculated (Fig. 6B). The expected mean rankings and SUCRA values of each pharmacological agent are presented in Fig. 7B. According to the SUCRA value, the incidence of POD was lower in the order of the Prop + AAP (85.9\%), followed by Keta + Dexm (83.2\%), Gaba (82.2\%), and Diaz + Flun + Pethi $(79.7 \%)$.

\section{Cardiac surgery}

A total of 19 studies (15,090 patients) were analyzed. The pooled overall incidence of POD after cardiac surgery was $15.4 \%$ (95\% CI: $12.8 \%$ to $18.4 \%, \mathrm{P}_{\text {chi }}{ }^{2}<0.001, \mathrm{I}^{2}=89.8 \%$ ).

The network plot of all eligible comparisons for this endpoint is depicted in Fig. 2C.

Although all 13 management modalities (nodes) were connected to the network, three comparisons (Cont, Dexm, Prop) were compared directly to the other 10 nodes.

The evaluation of the network inconsistency using the design-by-treatment interaction model suggested no significant inconsistency $\left(\chi^{2}[2]=4.12, \mathrm{P}=0.128\right)$. Of the five closed loops in the network of the comparison of postoperative delirium, two loops (Dexm-Dexm + AAP-Prop + AAP [01-04-05] [70] and Pro-Dexm + AAP-Prop + AAP [03-04-05] [70]) were formed only by multi-arm trials. Thus, local inconsistency was evaluated in three loops. There was no significance in the local inconsistency between the direct and indirect point estimates (Fig. 3C).

None of the regimens showed a lower incidence of POD than Cont only in terms of both 95\% CI and 95\% PrIs (Fig. 4C). The rankogram showed that Prop + AAP and Keta had the lowest incidence of POD (Fig. 5C). The cumulative ranking plot was drawn, and the SUCRA probabilities of the different pharmacological interventions for the POD were calculated (Fig. 6C). The expected mean rankings and SUCRA values of each pharmacological intervention are 

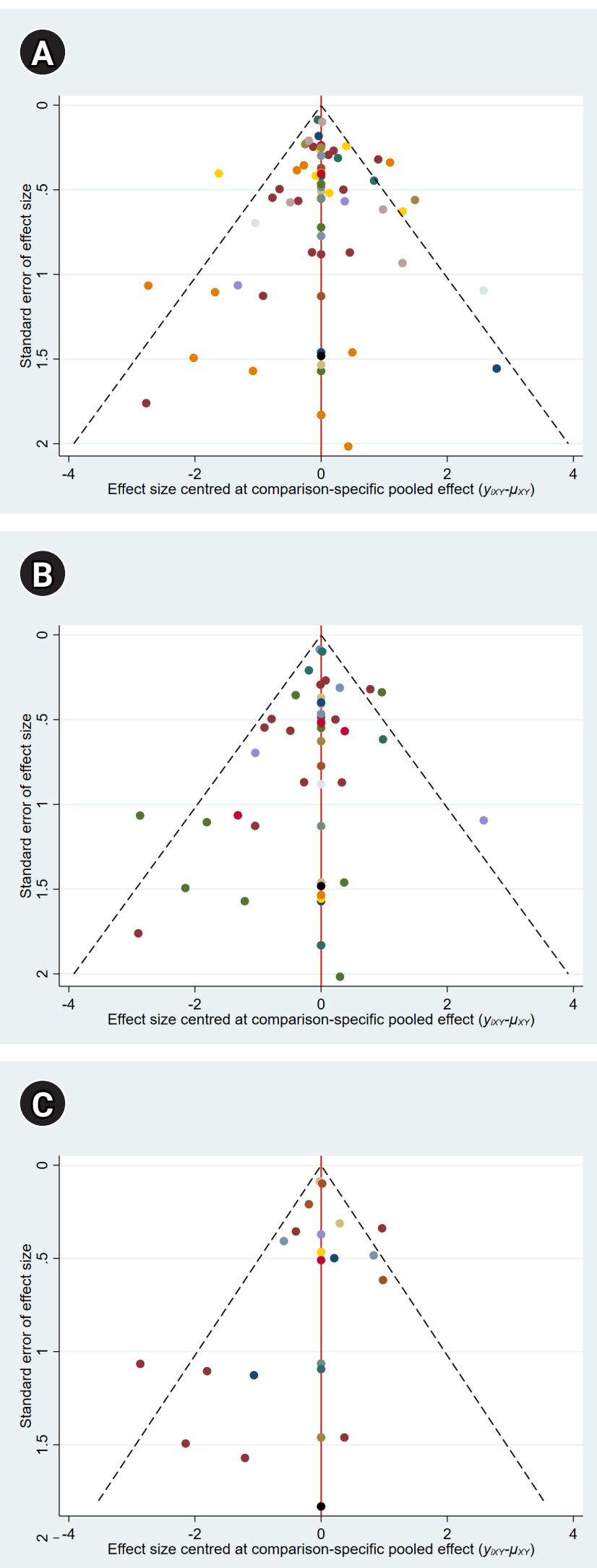

Fig. 8. Comparison-adjusted funnel plot. presented in Fig. 7C. According to the SUCRA value, the incidence of POD was lower in the order of Keta (87.1\%), Prop + AAP (86.0\%), followed by Dexm + AAP (66.3\%). The comparison-adjusted funnel plots showed that the funnel plots were symmetrical around the zero line, which suggested a less likely publication bias (Fig. 8C).

\section{Quality of evidence}

Three outcomes were evaluated using the GRADE system. For each outcome, the qualities of inconsistency, indirectness, imprecision and publication bias were assessed as not serious, but qualities of risk of bias were assessed as serious. Thus, the overall quality of evidence for each outcome was downgraded and rated as moderate (Table 3).

\section{DISCUSSION}

There are various pharmacological interventions to prevent POD. We performed a network meta-analysis to compare the effectiveness of reported pharmacological interventions. In our study, the incidence of POD was decreased in the following order: Prop + AAP, Keta + Dexm, Gaba, and Diaz + Flun + Pethi after all types of anesthesia; Prop + AAP, Keta +Dexm, Gaba, and Diaz + Flun + Pethi after general anesthesia; and Keta, Prop + AAP, and Dexm + AAP after cardiac surgery. However, only the Dexm group showed a statistically lower incidence of POD compared with the control group after all types of anesthesia and after general anesthesia.

In our study, there was a synergistic effect when Prop was added to AAP. Although Prop + AAP failed to show statistical significance, Prop + AAP was ranked the most effective pharmacological intervention with a low incidence of POD after all types of anesthesia and after general anesthesia. Prop is a short-acting, intravenous sedative-hypnotic agent commonly used for general anesthesia and sedation. It has also been used to control other conditions such as chemotherapy-induced emesis, as an antipruritic in patients with intractable pruritus due to liver disease, as an adjuvant in alcohol withdrawal syndrome, and to treat status epilepticus and severe refractory delirium. Prop has been recently shown to have a long-term neuroprotective effect and CNS inhibition effect [78-80]. AAP is commonly used as an adjuvant analgesic. Some prior studies have indicated that AAP reduces opioid consumption and inflammation. Recently, AAP has been shown to confer central 
Table 3. The GRADE Evidence Quality for Post-operative Delirium

\begin{tabular}{|c|c|c|c|c|c|c|c|}
\hline \multirow{2}{*}{ Type } & \multirow{2}{*}{ No. of studies } & \multicolumn{5}{|c|}{ Quality assessment } & \multirow{2}{*}{ Quality } \\
\hline & & Risk of bias & Inconsistency & Indirectness & Imprecision & Publication bias & \\
\hline All type of anesthesia & 51 & Serious & Not serious & Not serious & Serious & None & $\begin{array}{l}\oplus \oplus \oplus \bigcirc \\
\text { Moderate }\end{array}$ \\
\hline General Anesthesia & 35 & Serious & Not serious & Not serious & Serious & None & $\begin{array}{l}\oplus \oplus \oplus \bigcirc \\
\text { Moderate }\end{array}$ \\
\hline Cardiac surgery & 19 & Serious & Not serious & Not serious & Serious & None & $\begin{array}{l}\oplus \oplus \oplus \bigcirc \\
\text { Moderate }\end{array}$ \\
\hline
\end{tabular}

GRADE: grading of recommendations, assessment, development, and evaluation.

analgesic properties. This makes it likely that AAP would reduce delirium. Despite these properties, IV AAP has not been studied in the context of delirium prevention $[81,82]$. In our study, a synergistic effect occurred as the neuroprotective effect of Prop was added to the POD prevention effect of AAP.

Dexm, a selective $\alpha_{2}$-adrenergic agonist, has a strong modulating effect on the activity of the sympathetic system and is increasingly used as a sedative and an adjuvant anesthetic during surgery. Dexm binds to $\alpha_{2}$-receptors present in both the central and peripheral nervous systems [83-86]. Meanwhile, Dexm inhibits the release of norepinephrine and sympathetic activity. In our study, Dexm reduced POD after all types of anesthesia and after general anesthesia compared with the control group. These results are in close agreement with a previous report by $\mathrm{Al} \mathrm{Tmimi}$ et al. [13] and Shen et al. [14] in which the risks of POD were decreased in elderly patients after non-cardiac surgery.

Several mechanisms have been suggested to explain how Dexm reduces the incidence of POD after surgery and anesthesia. First, because of its highly selective and specific $\alpha_{2}$-adrenergic agonistic characteristics, Dexm reduces the amount of other sedatives and opioids used during surgery, which may cause POD development and prolongation $[40,51]$. Second, Dexm attenuates the immune cascade and inflammatory mediators, consequently relieving inflammatory response [87], which is associated with POD. Third, Dexm induces a near-natural sleep-like sedative pattern, which might help to reduce the risk of delirium significantly. In addition, Dexm has been suggested as a neuroprotectant during mechanical ventilation by reducing cerebral blood and cerebral perfusion pressure [88-90].

Keta, which is ineffective with monotherapy, when combined with Dexm becomes the most effective modality. Several reports in the past few years have evaluated Keta for the treatment of hyperactive delirium. Keta offers a po- tential option for treating difficult to manage hyperactive delirium [91]. Moreover, Keta is an NMDA receptor antagonist, which reduces post-ischemic neuronal cell loss in the cortex and improves neurological outcome after cerebral ischemia $[92,93]$. Thus, Keta may produce a prolonged effect on postoperative neurocognitive function by causing a "preconditioning-like" effect through the temporary inactivation of NMDA receptors, thereby rendering these receptors less susceptible to subsequent activation by ischemia and reperfusion injury. However, this intriguing hypothesis has not been formally tested. Keta may also confer neuroprotection by suppressing the inflammatory response after surgery $[71,94]$.

For quality of life, which has recently attracted attention, postoperative complications of surgical patients should be prevented and treated appropriately. Among complications, POD can directly or indirectly increase postoperative morbidity and mortality in elderly patients. Delirium is not always a transient disorder; in some cases, it may be accompanied by subtle structural brain damage, leading to permanent cognitive impairment. Therefore, there is growing interest in proper preventive methods $[62,68]$.

In our study, we focused on the incidence of POD, preventive effect of interventions, and collected pharmacological intervention data. There have been a number of preventative methods introduced in other studies, but their efficacy has not been properly compared. To compensate for this, our NMA including various pharmacological interventions. Throughout this study, we attempted to identify the most effective prevention of POD.

There are several limitations in this study. First, as with all meta-analyses, there were clinical and methodological heterogeneities regarding administration timing (for example, preoperative or intraoperative or postoperative), method (for example, bolus or continuous infusion) and dose spectrum of pharmacological interventions, and assessor of POD and assessment tool of POD. Second, in our study, 
incidence of POD was used as an indicator of prevention. However, to reduce morbidity and mortality associated with POD, it is also important to reduce the severity and duration of POD. Therefore, further studies should be conducted to evaluate the effects on the severity and duration of POD. Third, the most efficacious modalities determined in the current NMA were documented to be effective in a limited number of clinical trials. Further, as our NMA was based on various single-center small-scale trials, a risk of overestimation or underestimation of true treatment effects or lack of power to discriminate the effectiveness of pharmacological interventions may be present. Therefore, further large-scale RCTs with the qualified protocol should be conducted in the future to encompass different pharmacological interventions and substantiate our findings.

Despite these limitations, the current NMA has several strengths compared to previous NMAs. First, a rigorous methodology based on a published, pre-planned protocol to provide evidence of pharmacological interventions to prevent POD was used. Second, inconsistencies among the enrolled studies were not significant, and publication bias of the enrolled studies was minimal. Third, most enrolled studies exhibited a low risk of bias, except for bias from the randomization process and bias due to deviations from intended intervention domains.

In conclusion, the NMA performed in this study has strength and meaning for comparing pharmacological interventions in the clinical efficacy of preventing POD. Dexm showed a significant decrease in the incidence of POD compared with the control group. The combination of Prop and AAP and the combination of Keta and Dexm seemed to be effective in preventing POD. However, further studies are needed to determine the optimal pharmacological intervention to prevent POD.

\section{SUPPLEMENTARY MATERIALS}

Supplementary data including search terms used for MEDLINE and EMBASE can be found online at https://doi. org/10.17085/apm.20079

\section{ACKNOWLEDGEMENTS}

This research was supported by the Basic Science Research Program through the National Research Foundation of Korea (NRF) funded by the Ministry of Education, Science and Technology (2018R1A2A2A05021467).

\section{CONFLICTS OF INTEREST}

No potential conflict of interest relevant to this article was reported.

\section{AUTHOR CONTRIBUTIONS}

Conceptualization: Jun Mo Lee, Eun Jin Ahn, Geun Joo Choi, Hyun Kang. Data curation: Jun Mo Lee, Hyun Kang. Formal analysis: Ye Jin Cho, Hyun Kang. Funding acquisition: Hyun Kang. Methodology: Geun Joo Choi, Hyun Kang. Project administration: Jun Mo Lee, Ye Jin Cho, Hyun Kang. Visualization: Ye Jin Cho, Geun Joo Choi, Hyun Kang. Writing - original draft: Jun Mo Lee, Ye Jin Cho, Geun Joo Choi, Hyun Kang. Writing - review \& editing: Jun Mo Lee, Eun Jin Ahn, Hyun Kang. Investigation: Jun Mo Lee, Ye Jin Cho, Eun Jin Ahn, Hyun Kang. Resources: Jun Mo Lee, Hyun Kang. Software: Hyun Kang. Supervision: Eun Jin Ahn, Geun Joo Choi, Hyun Kang. Validation: Jun Mo Lee, Ye Jin Cho, Eun Jin Ahn, Hyun Kang.

\section{ORCID}

Jun Mo Lee, https://orcid.org/0000-0001-5607-7232

Ye Jin Cho, https://orcid.org/0000-0002-9138-6126

Eun Jin Ahn, https://orcid.org/0000-0001-6321-5285

Geun Joo Choi, https://orcid.org/0000-0002-4653-4193

Hyun Kang, https://orcid.org/0000-0003-2844-5880

\section{REFERENCES}

1. Li YN, Zhang Q, Yin CP, Guo YY, Huo SP, Wang L, et al. Effects of nimodipine on postoperative delirium in elderly under general anesthesia: a prospective, randomized, controlled clinical trial. Medicine (Baltimore) 2017; 96: e6849.

2. Liu Y, Ma L, Gao M, Guo W, Ma Y. Dexmedetomidine reduces postoperative delirium after joint replacement in elderly patients with mild cognitive impairment. Aging Clin Exp Res 2016; 28: 729-36.

3. American Geriatrics Society Expert Panel on Postoperative Delirium in Older Adults. Postoperative delirium in older adults: best practice statement from the American Geriatrics Society. J Am Coll Surg 2015 220: 136-48.e1.

4. Maldonado JR, Wysong A, van der Starre PJ, Block T, Miller C, Reitz BA. Dexmedetomidine and the reduction of postoperative delirium after cardiac surgery. Psychosomatics 2009; 50: 206-17. 
5. Parikh SS, Chung F. Postoperative delirium in the elderly. Anesth Analg 1995; 80: 1223-32.

6. He F, Shen L, Zhong J. A study of dexmedetomidine in the prevention of postoperative delirium in elderly patients after vertebral osteotomy. Int J Clin Exp Med 2018; 11: 4984-90.

7. Whitlock EL, Vannucci A, Avidan MS. Postoperative delirium. Minerva Anestesiol 2011; 77: 448-56.

8. Reddy SV, Irkal JN, Srinivasamurthy A. Postoperative delirium in elderly citizens and current practice. J Anaesthesiol Clin Pharmacol 2017; 33: 291-9.

9. Janssen TL, Alberts AR, Hooft L, Mattace-Raso F, Mosk CA, van der Laan L. Prevention of postoperative delirium in elderly patients planned for elective surgery: systematic review and meta-analysis. Clin Interv Aging 2019; 14: 1095-117.

10. Liu Y, Li XJ, Liang Y, Kang Y. Pharmacological prevention of postoperative delirium: a systematic review and meta-analysis of randomized controlled trials. Evid Based Complement Alternat Med 2019; 2019: 9607129.

11. Nazemi AK, Gowd AK, Carmouche JJ, Kates SL, Albert TJ, Behrend CJ. Prevention and management of postoperative delirium in elderly patients following elective spinal surgery. Clin Spine Surg 2017; 30: 112-9.

12. Zhang H, Lu Y, Liu M, Zou Z, Wang L, Xu FY, et al. Strategies for prevention of postoperative delirium: a systematic review and meta-analysis of randomized trials. Crit Care 2013; 17: R47.

13. Al Tmimi L, Verbrugghe P, Van de Velde M, Meuris B, Meyfroidt G, Milisen K, et al. Intraoperative xenon for prevention of delirium after on-pump cardiac surgery: a randomised, observer-blind, controlled clinical trial. Br J Anaesth. 2020. doi: 10. 1016/j.bja.2019.11.037. [Epub ahead of print].

14. Shen QH, Li HF, Zhou XY, Yuan XZ. Dexmedetomidine in the prevention of postoperative delirium in elderly patients following non-cardiac surgery: a systematic review and meta-analysis. Clin Exp Pharmacol Physiol 2020; 47: 1333-41.

15. Cui Y, Li G, Cao R, Luan L, Kla KM. The effect of perioperative anesthetics for prevention of postoperative delirium on general anesthesia: a network meta-analysis. J Clin Anesth 2020; 59: 89-98.

16.; PRISMA-P Group. Preferred reporting items for systematic review and meta-analysis protocols (PRISMA-P) 2015: elaboration and explanation. BMJ 2015; 350: g7647.

17. Higgins JPT, Thomas J, Chandler J, Cumpston M, Li T, Page M, et al. Cochrane handbook for systematic reviews of interventions. 2nd ed. Hoboken, Wiley-Blackwell. 2019.

18. Cornell JE. The PRISMA extension for network meta-analysis: bringing clarity and guidance to the reporting of systematic reviews incorporating network meta-analyses. Ann Intern Med
2015; 162: 797-8.

19. Viera AJ, Garrett JM. Understanding interobserver agreement: the kappa statistic. Fam Med 2005; 37: 360-3.

20. Higgins JPT, Savović J, Page MJ, Elbers RG, Sterne JAC. Assessing risk of bias in a randomized trial. In: Cochrane handbook for systematic reviews of interventions. 2nd ed. Edited by Higgins JPT, Thomas J, Chandler J, Cumpston M, Li T, Page M, et al.: Hoboken, Wiley-Blackwell. 2019.

21. Chaimani A, Higgins JP, Mavridis D, Spyridonos P, Salanti G. Graphical tools for network meta-analysis in STATA. PLoS One 2013; 8: e76654.

22. White IR, Barrett JK, Jackson D, Higgins JP. Consistency and inconsistency in network meta-analysis: model estimation using multivariate meta-regression. Res Synth Methods 2012; 3: 11125.

23. Salanti G, Ades AE, Ioannidis JP. Graphical methods and numerical summaries for presenting results from multiple-treatment meta-analysis: an overview and tutorial. J Clin Epidemiol 2011; 64: 163-71.

24. Atkins D, Best D, Briss PA, Eccles M, Falck-Ytter Y, Flottorp S, et al.; GRADE Working Group. Grading quality of evidence and strength of recommendations. BMJ 2004; 328: 1490.

25. O'Gara B, Marcantonio ER, Pascual-Leone A, Shaefi S, Mueller A, Banner-Goodspeed V, et al. Prevention of early postoperative decline (PEaPoD): protocol for a randomized, controlled feasibility trial. Trials 2018; 19: 676.

26. Shankar P, Mueller A, Packiasabapathy S, Gasangwa D, Patxot M, O'Gara B, et al. Dexmedetomidine and intravenous acetaminophen for the prevention of postoperative delirium following cardiac surgery (DEXACET trial): protocol for a prospective randomized controlled trial. Trials 2018; 19: 326.

27. Sánchez A, Thomas C, Deeken F, Wagner S, Klöppel S, Kentischer F, et al.; PAWEL Study group. Patient safety, cost-effectiveness, and quality of life: reduction of delirium risk and postoperative cognitive dysfunction after elective procedures in older adults-study protocol for a stepped-wedge cluster randomized trial (PAWEL Study). Trials 2019; 20: 71.

28. Farlinger C, Clarke H, Wong CL. Perioperative pregabalin and delirium following total hip arthroplasty: a post hoc analysis of a double-blind randomized placebo-controlled trial. Can J Anaesth 2018; 65: 1269-70.

29. Joys S, Samra T, Kumar V, Mohanty M, Sodhi HBS, Mahajan S, et al. Comparison of postoperative delirium in patients anesthetized with isoflurane versus desflurane during spinal surgery: a prospective randomized controlled trial. Surg Neurol Int 2019; 10: 226.

30. Wang X, Wang Y, Hu Y, Wang L, Zhao W, Wei L, et al. Effect of 
flurbiprofen axetil on postoperative delirium for elderly patients. Brain Behav 2019; 9: e01290.

31. Shehabi Y, Grant P, Wolfenden H, Hammond N, Bass F, Campbell M, et al. Prevalence of delirium with dexmedetomidine compared with morphine based therapy after cardiac surgery: a randomized controlled trial (DEXmedetomidine COmpared to Morphine-DEXCOM Study). Anesthesiology 2009; 111: 1075-84.

32. Royse CF, Saager L, Whitlock R, Ou-Young J, Royse A, Vincent J, et al. Impact of methylprednisolone on postoperative quality of recovery and delirium in the steroids in cardiac surgery trial: a randomized, double-blind, placebo-controlled substudy. Anesthesiology 2017; 126: 223-33.

33. Djaiani G, Silverton N, Fedorko L, Carroll J, Styra R, Rao V, et al. Dexmedetomidine versus propofol sedation reduces delirium after cardiac surgery: a randomized controlled trial. Anesthesiology 2016; 124: 362-8.

34. Whitlock RP, Devereaux PJ, Teoh KH, Lamy A, Vincent J, Pogue J, et al.; SIRS Investigators. Methylprednisolone in patients undergoing cardiopulmonary bypass (SIRS): a randomised, double-blind, placebo-controlled trial. Lancet 2015; 386: 1243-53.

35. Li X, Yang J, Nie XL, Zhang Y, Li XY, Li LH, et al. Impact of dexmedetomidine on the incidence of delirium in elderly patients after cardiac surgery: a randomized controlled trial. PLoS One 2017; 12: e0170757.

36. Su X, Meng ZT, Wu XH, Cui F, Li HL, Wang DX, et al. Dexmedetomidine for prevention of delirium in elderly patients after non-cardiac surgery: a randomised, double-blind, placebo-controlled trial. Lancet 2016; 388: 1893-902.

37. Wang W, Li HL, Wang DX, Zhu X, Li SL, Yao GQ, et al. Haloperidol prophylaxis decreases delirium incidence in elderly patients after noncardiac surgery: a randomized controlled trial*. Crit Care Med 2012; 40: 731-9.

38. Yang X, Li Z, Gao C, Liu R. Effect of dexmedetomidine on preventing agitation and delirium after microvascular free flap surgery: a randomized, double-blind, control study. J Oral Maxillofac Surg 2015; 73: 1065-72.

39. Ma P, Piao M, Wang Y, Ma H, Feng C. Influence of dexmedetomidine and sub-anesthetic dose of ketamine on postoperative delirium in elderly orthopedic patients under total intravenous anesthesia. J Jilin Univ Med Ed 2013; 39: 128-32.

40. Xuan Y, Fan R, Chen J, Wang Y, Wu J, Yang J, et al. Effects of dexmedetomidine for postoperative delirium after joint replacement in elderly patients: a randomized, double-blind, and placebo-controlled trial. Int J Clin Exp Med 2018; 11: 13147-57.

41. Huyan T, Hu X, Peng H, Zhu Z, Li Q, Zhang W. Perioperative dexmedetomidine reduces delirium in elderly patients after lung cancer surgery. Psychiatr Danub 2019; 31: 95-101.

42. Shi C, Jin J, Qiao L, Li T, Ma J, Ma Z. Effect of perioperative administration of dexmedetomidine on delirium after cardiac surgery in elderly patients: a double-blinded, multi-center, randomized study. Clin Interv Aging 2019; 14: 571-5.

43. Liu X, Zhang K, Wang W, Xie G, Fang X. Dexmedetomidine sedation reduces atrial fibrillation after cardiac surgery compared to propofol: a randomized controlled trial. Crit Care 2016; 20: 298.

44. Mei B, Meng G, Xu G, Cheng X, Chen S, Zhang Y, et al. Intraoperative sedation with dexmedetomidine is superior to propofol for elderly patients undergoing hip arthroplasty: a prospective randomized controlled study. Clin J Pain 2018; 34: 811-7.

45. Guo Y, Sun LL, Chen ZF, Li QF, Jiang H. [Preventive effect of dexmedetomidine on postoperative delirium in elderly patients with oral cancer]. Shanghai Kou Qiang Yi Xue 2015; 24: 236-9. Chinese.

46. Mu DL, Zhang DZ, Wang DX, Wang G, Li CJ, Meng ZT, et al. Parecoxib supplementation to morphine analgesia decreases incidence of delirium in elderly patients after hip or knee replacement surgery: a randomized controlled trial. Anesth Analg 2017; 124: 1992-2000.

47. Liu X, Zhang K, Wang W, Xie G, Cheng B, Wang Y, et al. Dexmedetomidine versus propofol sedation improves sublingual microcirculation after cardiac surgery: a randomized controlled trial. J Cardiothorac Vasc Anesth 2016; 30: 1509-15.

48. Clemmesen CG, Lunn TH, Kristensen MT, Palm H, Foss NB. Effect of a single pre-operative $125 \mathrm{mg}$ dose of methylprednisolone on postoperative delirium in hip fracture patients; a randomised, double-blind, placebo-controlled trial. Anaesthesia 2018; 73: 1353-60.

49. Papadopoulos G, Pouangare M, Papathanakos G, Arnaoutoglou E, Petrou A, Tzimas P. The effect of ondansetron on postoperative delirium and cognitive function in aged orthopedic patients. Minerva Anestesiol 2014; 80: 444-51.

50. Priye S, Jagannath S, Singh D, Shivaprakash S, Reddy DP. Dexmedetomidine as an adjunct in postoperative analgesia following cardiac surgery: a randomized, double-blind study. Saudi J Anaesth 2015; 9: 353-8.

51. Sheikh TA, Dar BA, Akhter N, Ahmad N. A comparative study evaluating effects of intravenous sedation by dexmedetomidine and propofol on patient hemodynamics and postoperative outcomes in cardiac surgery. Anesth Essays Res 2018; 12: 555-60.

52. Mardani D, Bigdelian H. Prophylaxis of dexamethasone protects patients from further post-operative delirium after cardiac surgery: a randomized trial. J Res Med Sci 2013; 18: 137-43. 
53. Mohammadi M, Ahmadi M, Khalili H, Cheraghchi H, Arbabi M. Cyproheptadine for the prevention of postoperative delirium: a pilot study. Ann Pharmacother 2016; 50: 180-7.

54. Fukata S, Kawabata Y, Fujisiro K, Katagawa Y, Kuroiwa K, Akiyama $\mathrm{H}$, et al. Haloperidol prophylaxis does not prevent postoperative delirium in elderly patients: a randomized, open-label prospective trial. Surg Today 2014; 44: 2305-13.

55. Kaneko T, Cai J, Ishikura T, Kobayashi M, Naka T, Kaibara N. Prophylactic consecutive administration of haloperidol can reduce the occurrence of postoperative delirium in gastrointestinal surgery. Yonago Acta Medica 1999; 42: 179-84.

56. Sugano N, Aoyama T, Sato T, Kamiya M, Amano S, Yamamoto N, et al. Randomized phase II study of TJ-54 (Yokukansan) for postoperative delirium in gastrointestinal and lung malignancy patients. Mol Clin Oncol 2017; 7: 569-73.

57. Aizawa K, Kanai T, Saikawa Y, Takabayashi T, Kawano Y, Miyazawa $\mathrm{N}$, et al. A novel approach to the prevention of postoperative delirium in the elderly after gastrointestinal surgery. Surg Today 2002; 32: 310-4.

58. de Jonghe A, van Munster BC, Goslings JC, Kloen P, van Rees C, Wolvius R, et al; Amsterdam Delirium Study Group. Effect of melatonin on incidence of delirium among patients with hip fracture: a multicentre, double-blind randomized controlled trial. CMAJ 2014; 186: E547-56.

59. Dieleman JM, Nierich AP, Rosseel PM, van der Maaten JM, Hofland J, Diephuis JC, et al.; Dexamethasone for Cardiac Surgery (DECS) Study Group. Intraoperative high-dose dexamethasone for cardiac surgery: a randomized controlled trial. JAMA 2012; 308: 1761-7.

60. Kalisvaart KJ, de Jonghe JF, Bogaards MJ, Vreeswijk R, Egberts TC, Burger BJ, et al. Haloperidol prophylaxis for elderly hip-surgery patients at risk for delirium: a randomized placebo-controlled study. J Am Geriatr Soc 2005; 53: 1658-66.

61. Sauër AM, Slooter AJ, Veldhuijzen DS, van Eijk MM, Devlin JW, van Dijk D. Intraoperative dexamethasone and delirium after cardiac surgery: a randomized clinical trial. Anesth Analg 2014; 119: 1046-52.

62. Park JB, Bang SH, Chee HK, Kim JS, Lee SA, Shin JK. Efficacy and safety of dexmedetomidine for postoperative delirium in adult cardiac surgery on cardiopulmonary bypass. Korean J Thorac Cardiovasc Surg 2014; 47: 249-54.

63. Lee C, Lee CH, Lee G, Lee M, Hwang J. The effect of the timing and dose of dexmedetomidine on postoperative delirium in elderly patients after laparoscopic major non-cardiac surgery: a double blind randomized controlled study. J Clin Anesth 2018; 47: 27-32.

64. Gamberini M, Bolliger D, Lurati Buse GA, Burkhart CS, Grapow
M, Gagneux A, et al. Rivastigmine for the prevention of postoperative delirium in elderly patients undergoing elective cardiac surgery--a randomized controlled trial. Crit Care Med 2009; 37: $1762-8$.

65. Wu CY, Lu YF, Wang ML, Chen JS, Hsu YC, Yang FS, et al. Effects of dexmedetomidine infusion on inflammatory responses and injury of lung tidal volume changes during one-lung ventilation in thoracoscopic surgery: a randomized controlled trial. Mediators Inflamm 2018; 2018: 2575910.

66. Chang YF, Chao A, Shih PY, Hsu YC, Lee CT, Tien YW, et al.; NTUH Center of Microcirculation Medical Research (NCMMR). Comparison of dexmedetomidine versus propofol on hemodynamics in surgical critically ill patients. J Surg Res 2018; 228: 194-200.

67. Prakanrattana U, Prapaitrakool S. Efficacy of risperidone for prevention of postoperative delirium in cardiac surgery. Anaesth Intensive Care 2007; 35: 714-9.

68. Sampson EL, Raven PR, Ndhlovu PN, Vallance A, Garlick N, Watts J, et al. A randomized, double-blind, placebo-controlled trial of donepezil hydrochloride (Aricept) for reducing the incidence of postoperative delirium after elective total hip replacement. Int J Geriatr Psychiatry 2007; 22: 343-9.

69. Deiner S, Luo X, Lin HM, Sessler DI, Saager L, Sieber FE, et al. Intraoperative infusion of dexmedetomidine for prevention of postoperative delirium and cognitive dysfunction in elderly patients undergoing major elective noncardiac surgery: a randomized clinical trial. JAMA Surg 2017; 152: e171505.

70. Susheela AT, Packiasabapathy S, Gasangwa DV, Patxot M, O'Neal J, Marcantonio E, et al. The use of dexmedetomidine and intravenous acetaminophen for the prevention of postoperative delirium in cardiac surgery patients over 60 years of age: a pilot study. F1000Res 2017; 6: 1842.

71. Hudetz JA, Patterson KM, Iqbal Z, Gandhi SD, Byrne AJ, Hudetz AG, et al. Ketamine attenuates delirium after cardiac surgery with cardiopulmonary bypass. J Cardiothorac Vasc Anesth 2009; 23: 651-7.

72. Larsen KA, Kelly SE, Stern TA, Bode RH Jr, Price LL, Hunter DJ, et al. Administration of olanzapine to prevent postoperative delirium in elderly joint-replacement patients: a randomized, controlled trial. Psychosomatics 2010; 51: 409-18.

73. Leung JM, Sands LP, Chen N, Ames C, Berven S, Bozic K, et al.; Perioperative Medicine Research Group. Perioperative gabapentin does not reduce postoperative delirium in older surgical patients: a randomized clinical trial. Anesthesiology 2017; 127: 633-44.

74. Robinson TN, Dunn CL, Adams JC, Hawkins CL, Tran ZV, Raeburn CD, et al. Tryptophan supplementation and postopera- 
tive delirium--a randomized controlled trial. J Am Geriatr Soc 2014; 62: 1764-71.

75. Liptzin B, Laki A, Garb JL, Fingeroth R, Krushell R. Donepezil in the prevention and treatment of post-surgical delirium. Am J Geriatr Psychiatry 2005; 13: 1100-6.

76. Leung JM, Sands LP, Rico M, Petersen KL, Rowbotham MC, Dahl JB, et al. Pilot clinical trial of gabapentin to decrease postoperative delirium in older patients. Neurology 2006; 67: 12513.

77. Sultan SS. Assessment of role of perioperative melatonin in prevention and treatment of postoperative delirium after hip arthroplasty under spinal anesthesia in the elderly. Saudi J Anaesth 2010; 4: 169-73.

78. Saporito A, Sturini E. Incidence of postoperative delirium is high even in a population without known risk factors. J Anesth 2014; 28: 198-201.

79. Brown KE, Mirrakhimov AE, Yeddula K, Kwatra MM. Propofol and the risk of delirium: exploring the anticholinergic properties of propofol. Med Hypotheses 2013; 81: 536-9.

80. Covarrubias-Gómez A, López Collada-Estrada M. Propofol-based palliative sedation to treat antipsychotic-resistant agitated delirium. J Pain Palliat Care Pharmacother 2017; 31: 190-4.

81. Subramaniam B, Shankar P, Shaefi S, Mueller A, O'Gara B, Banner-Goodspeed V, et al. Effect of intravenous acetaminophen vs placebo combined with propofol or dexmedetomidine on postoperative delirium among older patients following cardiac surgery: the DEXACET randomized clinical trial. JAMA 2019; 321: 686-96.

82. Farkas J. PULMCrit- DEXACET: four grams of acetaminophen a day keeps the delirium away? PULMCrit [serial on the Internet]. 2019 Mar [cited 2020 Apr]. Available from https://emcrit. org/pulmcrit/dexacet/.

83. Cai Y, Xu H, Yan J, Zhang L, Lu Y. Molecular targets and mechanism of action of dexmedetomidine in treatment of ischemia/ reperfusion injury. Mol Med Rep 2014; 9: 1542-50.

84. Sottas CE, Anderson BJ. Dexmedetomidine: the new all-in-one drug in paediatric anaesthesia? Curr Opin Anaesthesiol 2017;
30: 441-51.

85. Fan H, Zhao Y, Sun M, Ye JH, Chen GD, Zhu JH. Dexmedetomidine based sedation for post-surgery critically ill adults: a meta-analysis of randomized controlled trials. Iran J Public Health 2017; 46: 1611-22.

86. Yu X, Franks NP, Wisden W. Sleep and sedative states induced by targeting the histamine and noradrenergic systems. Front Neural Circuits 2018; 12: 4.

87. Sanders RD, Hussell T, Maze M. Sedation \& immunomodulation. Crit Care Clin 2009; 25: 551-70.

88. Bekker A, Haile M, Kline R, Didehvar S, Babu R, Martiniuk F, et al. The effect of intraoperative infusion of dexmedetomidine on the quality of recovery after major spinal surgery. J Neurosurg Anesthesiol 2013; 25: 16-24.

89. Zhang X, Wang J, Qian W, Zhao J, Sun L, Qian Y, et al. Dexmedetomidine inhibits tumor necrosis factor-alpha and interleukin 6 in lipopolysaccharide-stimulated astrocytes by suppression of c-Jun N-terminal kinases. Inflammation 2014; 37: 942-9.

90. Chen J, Yan J, Han X. Dexmedetomidine may benefit cognitive function after laparoscopic cholecystectomy in elderly patients. Exp Ther Med 2013; 5: 489-94.

91. Hayes BD. Ketamine for excited delirium syndrome. ALiEM [serial on the Internet]. 2015 May [cited 2020 Apr]. Available from https://www.aliem.com/ketamine-for-excited-delirium-syndrome/.

92. Reeker W, Werner C, Möllenberg O, Mielke L, Kochs E. Highdose S(+)-ketamine improves neurological outcome following incomplete cerebral ischemia in rats. Can J Anaesth 2000; 47: $572-8$.

93. Proescholdt M, Heimann A, Kempski O. Neuroprotection of $\mathrm{S}(+)$ ketamine isomer in global forebrain ischemia. Brain Res 2001; 904: 245-51.

94. Avidan MS, Maybrier HR, Abdallah AB, Jacobsohn E, Vlisides PE, Pryor KO, et al.; PODCAST Research Group. Intraoperative ketamine for prevention of postoperative delirium or pain after major surgery in older adults: an international, multicentre, double-blind, randomised clinical trial. Lancet 2017; 390: 26775 . 